\title{
Full colour for loop amplitudes in Yang-Mills theory
}

\author{
Alexander Ochirov ${ }^{a}$ and Ben Page ${ }^{b}$ \\ ${ }^{a}$ Higgs Centre for Theoretical Physics, School of Physics and Astronomy, \\ The University of Edinburgh, \\ Edinburgh EH9 3JZ, Scotland, U.K. \\ ${ }^{b}$ Albert-Ludwigs-Universität Freiburg, Physikalisches Institut, \\ D-79104 Freiburg, Germany \\ E-mail: alexander.ochirov@ed.ac.uk, ben.page@physik.uni-freiburg.de
}

ABStract: We present a general method to account for full colour dependence YangMills amplitudes at loop level. The method fits most naturally into the framework of multi-loop integrand reduction and in a nutshell amounts to consistently retaining the colour structures of the unitarity cuts from which the integrand is gradually constructed. This technique has already been used in the recent calculation of the two-loop five-gluon amplitude in pure Yang-Mills theory with all positive helicities, JHEP 10 (2015) 064. In this note, we give a careful exposition of the method and discuss its connection to looplevel Kleiss-Kuijf relations. We also explore its implications for cancellation of nontrivial symmetry factors at two loops. As an example of its generality, we show how it applies to the three-loop case in supersymmetric Yang-Mills case.

KeYwords: Scattering Amplitudes, Perturbative QCD

ARXIV EPRINT: 1612.04366 


\section{Contents}

1 Introduction 1

2 Trace-based colour decomposition $\quad 2$

2.1 Cuts and irreducible numerators 4

2.2 Colour ordering for irreducible numerators 5

2.3 Four-point two-loop example 8

3 DDM-based colour decomposition 11

$\begin{array}{lll}3.1 & \text { Kleiss-Kuijf relations for irreducible numerators } & 12\end{array}$

$\begin{array}{lll}3.2 & \text { One-loop case } & 14\end{array}$

$\begin{array}{lll}3.3 & \text { Two-loop case } & 16\end{array}$

$\begin{array}{lll}\text { 3.3.1 Two-loop symmetry factors } & 18\end{array}$

$\begin{array}{lll}\text { 3.3.2 Five-point two-loop amplitude } & 19\end{array}$

$\begin{array}{lll}3.4 & \text { Three-loop case } & 20\end{array}$

$\begin{array}{lll}3.4 .1 & \text { Four-point three-loop example } & 21\end{array}$

4 Summary and outlook $\quad 24$

$\begin{array}{ll}\text { A Bubble-insertion subtlety } & 25\end{array}$

B Check of four-point three-loop example 26

\section{Introduction}

With the increasing precision of measurements at the Large Hadron Collider, the accuracy of next-to-leading order (NLO) theoretical predictions calls for improvement. Making nextto-next-to-leading order (NNLO) computations as standard as the NLO ones constitutes a formidable challenge for theorists and is far from being achieved. Indeed, all the impressive achievements beyond NLO [1-24] are so far limited to processes with four or fewer coloured external states. Higher-multiplicity processes at NNLO seem to demand a similar leap in computational techniques, as already once happened at NLO, where the methods based on (generalised) unitarity cuts [25-31] eventually lead to the feasibility of one-loop calculations for up to seven external partons [32, 33].

Extending the NLO unitarity methods to higher loop orders is a challenge. Work in the direction of addressing this problem was first made in the context of maximal unitarity [3443], which seeks to directly reconstruct the coefficients of master integrals. An alternative approach [44-46] generalises the Ossola-Papadopoulos-Pittau method of integrand reduction [47] by making use of computational algebraic geometry [48-52]. The $D$-dimensional version of this method has been used to compute the most symmetric two-loop gluonic amplitudes - with all helicities positive (all-plus) - at five [53, 54] and six points [55]. 
Moreover, the successful integration of the planar five-gluon amplitude [56] has led [57, 58] to a direct unitarity calculation [59] of the polylogarithmic part of the $n$-point two-loop all-plus amplitude at leading colour.

In the calculation of a fully colour-dressed amplitude the number of loop-level partial amplitudes tends to grow quite fast [60-63]. That is why the full-colour integrand of the two-loop five-point all-plus amplitude was given by Badger, Mogull, O'Connell and one of the current authors in ref. [54] as a whole, rather than in terms of its partial amplitudes. In this note, we expand on the colour-decomposition method used in the calculation of ref. [54]. The method fits into the framework using multi-loop integrand reduction and, in a nutshell, amounts to a consistent way of retaining the colour factors of the generalised unitarity cuts, from which the irreducible numerators of the integrand are extracted. As explained in section 2, the irreducibility properties of the numerators and the top-down approach of gradual integrand construction guarantee that no contribution is double counted in the process. Once all nonzero irreducible numerators are known, the method provides a way to assemble the full-colour amplitude.

To illustrate the basic structure of the method, we begin in section 2 by applying it in the context of a trace-based colour decomposition that is conceptually simple. After careful consideration, one sees that this decomposition is redundant under Kleiss-Kuif relations [64]. In section 3 we proceed to show that this redundancy can easily be eliminated by applying the method to the colour decomposition of Del Duca, Dixon and Maltoni (DDM) $[65,66]$, thereby resulting in shorter expressions. Further we show that at one and two loops in this framework one can eliminate symmetry factors in a colour-ordered calculation. In order to aid understanding, we include in section 3 examples of the decomposition at one, two and three loops. All results of this paper trivially extend to Yang-Mills theories with supersymmetry or in higher dimensions.

\section{Trace-based colour decomposition}

In this section we describe the colour decomposition of a full purely gluonic amplitude at arbitrary loop order $L$, which follows from its construction from generalised unitarity cuts and naturally inherits the colour structures of the cuts.

In the unitarity method, a loop amplitude is fully constrained by a "spanning" set of generalised unitarity cuts [67] that are constructed out of $D$-dimensional tree amplitudes. The tree-level amplitudes contain both colour and kinematic information, and a standard way to separate these in the pure-gluon case is to reduce all the purely adjoint colour structures that appear in the Feynman diagrams into combinations of fundamental traces:

$$
\mathcal{A}_{n}^{(0)}=g^{n-2} \sum_{\sigma \in S_{n} / D_{n}} T(\sigma(1), \sigma(2), \ldots, \sigma(n)) A(\sigma(1), \sigma(2), \ldots, \sigma(n)),
$$

where the sum is over $(n-1) ! / 2$ noncyclic reflection-inequivalent permutations applied to the standard ordering and the associated colour factors are

$$
T(1,2, \ldots, n) \equiv \operatorname{Tr}\left(T^{a_{1}} T^{a_{2}} \ldots T^{a_{n}}\right)+(-1)^{n} \operatorname{Tr}\left(T^{a_{n}} \ldots T^{a_{2}} T^{a_{1}}\right) .
$$




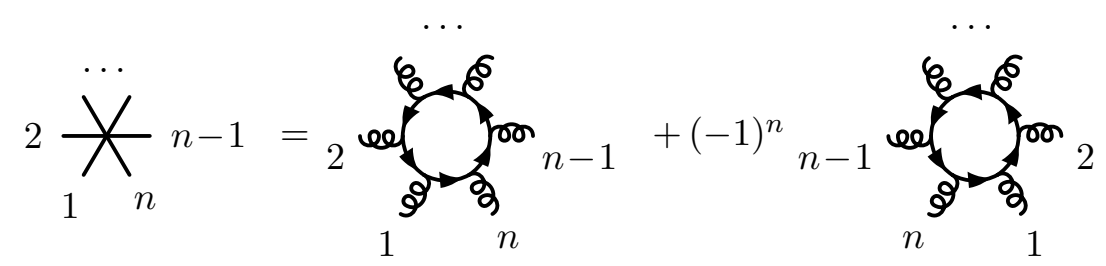

Figure 1. Graphic version of the definition $(2.2)$ for $T(1,2, \ldots, n)$. On the left-hand side it is depicted as an $n$-point vertex, since in section 2.1 these combine into trace-based colour factors for irreducible numerators.

For instance, in the three-gluon case the only element of $S_{3} / D_{3}$ is the identity permutation, and $T(1,2,3)$ coincides with the (imaginary) structure constant $\tilde{f}^{a_{1} a_{2} a_{3}}=\operatorname{Tr}\left(\left[T^{a_{1}}, T^{a_{2}}\right] T^{a_{3}}\right),{ }^{1}$ that corresponds to $A(1,2,3)$.

The tree-level colour decomposition (2.1) allows us to write a coloured unitarity cut, constructed out of $v$ tree amplitudes with arguments $\tau_{j}$, explicitly in terms of the constituent colour-ordered amplitudes:

$$
\operatorname{Cut}_{\mathcal{I}} \equiv \prod_{j=1}^{v} \mathcal{A}^{(0)}\left(\tau_{j}\right)=g^{n+2 L-2} \sum_{\substack{\sigma_{1} \in S_{\left|\tau_{1}\right|} / D_{\left|\tau_{1}\right|} \\ \sigma_{v} \in S_{\left|\tau_{v}\right|} / D_{\left|\tau_{v}\right|}}} \prod_{j=1}^{v} T\left(\sigma_{j}\left(\tau_{j}\right)\right) A\left(\sigma_{j}\left(\tau_{j}\right)\right) .
$$

The labelling that we use here refers to cuts by the sets of legs of their vertices, which are understood in the unordered sense for $\mathcal{I}=\left\{\tau_{1}, \ldots, \tau_{v}\right\}$. Now if we define a colour-ordered cut as constructed from colour-ordered tree amplitudes,

$$
\mathrm{Cut}_{i} \equiv \prod_{j=1}^{v} A\left(\sigma_{j}\right), \quad i \equiv\left\{\sigma_{1}, \ldots, \sigma_{v}\right\}
$$

where its label $i$ is the ordered analogue of $\mathcal{I}$, then it is natural to define the associated colour factor as

$$
T_{i} \equiv \prod_{j=1}^{v} T\left(\sigma_{j}\right), \quad i=\left\{\sigma_{1}, \ldots, \sigma_{v}\right\},
$$

so that the coloured cut is simply a linear combination of

$$
\begin{aligned}
\mathcal{C} \mathrm{ut}_{\mathcal{I}}=g^{n+2 L-2} & \sum_{i=\left\{\begin{array}{l}
\sigma_{1} \in S_{\left|\tau_{1}\right|} / D_{\left|\tau_{1}\right|} \\
\sigma_{v} \in S_{\left|\tau_{v}\right|} / D_{\left|\tau_{v}\right|} \mid
\end{array}\right.} T_{i} \mathrm{Cut}_{i} .
\end{aligned}
$$

In both cases, they include external particles or internal edges, corresponding to cut propagators. Moreover, we assume summation over the spins and colour indices of the internal edges, ${ }^{2}$ and this consequently applies to the cut colour factors $T_{i}$.

\footnotetext{
${ }^{1}$ We normalise the group generators to obey $\operatorname{Tr}\left(T^{a} T^{b}\right)=\delta^{a b}$ and $\left[T^{a}, T^{b}\right]=\tilde{f}^{a b c} T^{c}$.

${ }^{2}$ Working at the integrand level allows us to ignore the integration over the cut phase space in eq. (2.3).
} 


\subsection{Cuts and irreducible numerators}

Having defined natural colour factors for colour-ordered unitarity cuts, we now discuss a way to translate this to the colour decomposition of a loop amplitude. A modern constructive way to built a multi-loop amplitude from its unitarity cuts is the method of integrand reduction [44, 45, 47-49]. It uses computational algebraic geometry [48-52] to set up a bijection between each cut and its corresponding irreducible numerator,

$$
\operatorname{Cut}_{\mathcal{I}} \Leftrightarrow \tilde{\Delta}_{\mathcal{I}}
$$

such that the contribution of the latter to the loop integrand follows from the condition that the loop amplitude must satisfy correct unitarity cuts:

$$
\left.\mathcal{A}^{(L)}\right|_{\mathcal{I}}=\mathcal{C} u_{\mathcal{I}} \quad \Leftrightarrow \quad \int \frac{\mathrm{d}^{L D} \ell}{(2 \pi)^{L D}} \frac{\tilde{\Delta}_{\mathcal{I}}}{\prod_{j \in \mathcal{I}} D_{j}} \in \mathcal{A}^{(L)} .
$$

The key to this procedure is to define $\tilde{\Delta}_{\mathcal{I}}$ so that it is equivalent to the cut on the cut kinematics, but can also be continued off shell. That is, unlike the cut it is obtained from, $\tilde{\Delta}_{\mathcal{I}}$ is constructed in such a way that it can be evaluated for arbitrary phase space points, not just those satisfying the cut conditions. This procedure allows for a great degree of freedom and constructing continuations which simplify final expressions [54, 55] or take into account IBP relations [46] is an active topic of research. In full generality, $\tilde{\Delta}_{\mathcal{I}}$ is a function which lives in the finite-dimensional vector space of functions which, when evaluated on cut kinematics, span the space of all numerator functions allowed by theory power counting. Henceforth we leave the choice of basis implicit, but a useful example to keep in mind would be independent monomials of appropriate loop variables, such as irreducible scalar products (ISPs) of the type $\left(\ell_{i} \cdot p_{j}\right)$ that cannot be rewritten as a linear combination of the topology's propagators.

To define irreducible numerators, it is natural to start with the maximal cuts, i.e. with all the possible propagators cut. ${ }^{3}$ Therefore, on the maximal-cut kinematics, the bijection (2.7) is simply

$$
\tilde{\Delta}_{\mathcal{I}=\text { max.top. }}=\mathcal{C} \text { ut }_{\mathcal{I}=\text { max.top. }},
$$

from which the coordinates in the vector space are obtained. Then $\tilde{\Delta}_{\mathcal{I}=\text { max.top. becomes }}$ a well-defined function in the vector space away from the maximal-cut kinematics, despite the fact that eq. (2.9) stops being true, since the right-hand side is no longer defined.

At this point we can see that the colour decomposition of the maximal-topology numerator must correspond to that of the maximal cut, i.e.

$$
\begin{gathered}
\tilde{\Delta}_{\mathcal{I}=\text { max.top. }}=g^{n+2 L-2} \sum_{i=\left\{\begin{array}{l}
\sigma_{1} \in S_{\left|\tau_{1}\right|} / D_{\left|\tau_{1}\right|} \mid \\
\sigma_{v} \in S_{\left|\tau_{v}\right|} / D_{\left|\tau_{v}\right|}
\end{array}\right\}} T_{i} \Delta_{i},
\end{gathered}
$$

where $\Delta_{i}$ are colour-ordered irreducible numerators. The only fact that we implicitly use here is that for different orderings $\Delta_{i}$ corresponding to the same $\tilde{\Delta}_{\mathcal{I}}$ we choose the same

\footnotetext{
${ }^{3}$ In $D=4-2 \epsilon$ dimensions, a cut is maximal either for a trivalent topology with $n+3(L-1)$ loopdependent propagators or by fixing all the $4 L+L(L+1) / 2$ parameters of the loop-momentum space.
} 
basis of numerator function space. In fact, we are going to adhere to this choice throughout this paper. Moreover, the ordered topologies differing only by reflection of one or more of its vertices are considered identical (up to a possible sign), as implied by their reflectioninequivalent permutations sums.

Once the numerators for the maximal-level topologies are determined, one proceeds in the top-down approach, by gradually reducing the number of cut conditions and thereby descending to topologies with higher-point vertices. However, lower-level cuts are no longer polynomial functions, since they contain poles corresponding to higher-level cuts. In order to define the bijection (2.7) one needs to subtract those poles, with their residues already captured by the higher-level numerators:

$$
\tilde{\Delta}_{\mathcal{I}}=\mathcal{C u t}_{\mathcal{I}}-\sum_{\mathcal{J}>\mathcal{I}} \frac{\tilde{\Delta}_{\mathcal{J}}}{\prod_{l \in \mathcal{J} \backslash \mathcal{I}}\left(-i D_{l}\right)},
$$

where by $\mathcal{J}>\mathcal{I}$ we denote the topologies obtained by exposing loop-dependent propagators concealed inside the higher-point vertices of topology $\mathcal{I}$. These propagators are seen in the denominator, with the factors of $-i$ coming from the convention that a unitarity cut is obtained by replacing $i / D_{l} \rightarrow \delta\left(D_{l}\right)$ in a loop amplitude. (In practice, these can be reabsorbed into $\tilde{\Delta}_{\mathcal{I}}$.) Thanks to this level-subtraction procedure, the right-hand side of eq. (2.11), evaluated on $\mathcal{C}$ ut $_{\mathcal{I}}$-kinematics, can be used to resolve for the basis coefficients of the function $\tilde{\Delta}_{\mathcal{I}}$. Moreover, in this way $\tilde{\Delta}_{\mathcal{I}} / \prod_{l \in \mathcal{I}} D_{l}$ becomes the right integrand that one must add to the previously determined part of the loop amplitude to ensure that the latter satisfies $\mathcal{C u t}_{\mathcal{I}}$, as indicated by the relationship (2.8). The only subtlety here is that the cut topology $\mathcal{I}$ might have a nontrivial symmetry factor $S_{\mathcal{I}}$, in general equal to the number of ways of interchanging propagators or vertices without changing the topology. Taking this into account, as well as that cut diagrams have to be one-particle irreducible (1PI), the loop amplitude can be written as a sum over such diagrams,

$$
\mathcal{A}_{n}^{(L)}=\sum_{\mathcal{I} \in 1 \text { PI graphs }} \int \frac{\mathrm{d}^{L D} \ell}{(2 \pi)^{L D}} \frac{\tilde{\Delta}_{\mathcal{I}}}{S_{\mathcal{I}} \prod_{l \in \mathcal{I}}\left(-i D_{l}\right)} .
$$

With this representation of the amplitude, we observe that the routing of momentum used to define the irreducible numerator is now irrelevant as the numerators live under an integral sign. We will see in section 3 that in symmetric cases one encounters topologically equivalent terms with different loop-momentum routings. Due to the effective momentum rerouting invariance endowed by the integral sign, these terms are then seen to be equivalent, simplifying the resulting expression for the full-colour amplitude.

\subsection{Colour ordering for irreducible numerators}

So far we wrote the hierarchy subtraction (2.11) and the resulting amplitude (2.12) in terms of coloured objects, but it is not hard to colour-order them. Indeed, the hierarchy subtraction (2.11) is inductive and starts with the maximal-level topologies, for which we already have the trace-based colour decomposition (2.10). Its natural generalisation is 
simply

$$
\begin{gathered}
\tilde{\Delta}_{\mathcal{I}}=g^{n+2 L-2} \sum_{i=\left\{\begin{array}{l}
\sigma_{1} \in S_{\left|\tau_{1}\right|} / D_{\left|\tau_{1}\right|} \mid \\
\sigma_{v} \in S_{\left|\tau_{v}\right|} / D_{\left|\tau_{v}\right|}
\end{array}\right\}} T_{i} \Delta_{i} .
\end{gathered}
$$

One can see that this is true by considering the principle of eq. (2.11) applied to the cut colour decomposition (2.6). On the left-hand side of (2.13), one subtracts all poles from $\mathcal{C}$ ut $_{\mathcal{I}}$ by finding the coloured numerators associated to more constrained cuts, which one should here understand as factorisation limits of the original cut. The right-hand side of (2.13) is therefore found by considering the relevant factorisation limits of each colour-ordered cut. In effect, eq. (2.13) matches the colour decomposition (2.6) of the corresponding unitarity cut and the hierarchy subtraction (2.11) is then projected onto the colour-ordered numerators:

$$
\Delta_{i}=\mathrm{Cut}_{i}-\sum_{j>i} \frac{\Delta_{j}}{\prod_{l \in j \backslash i}\left(-i D_{l}\right)},
$$

where the " $j>i$ " sum goes over the ordered topologies obtained by exposing loop-dependent denominators inside the higher-point vertices of ordered topology $i$.

Let us illustrate this projection in more detail with a short example. Consider the following trace-basis colour decompositions of parent and child cuts:

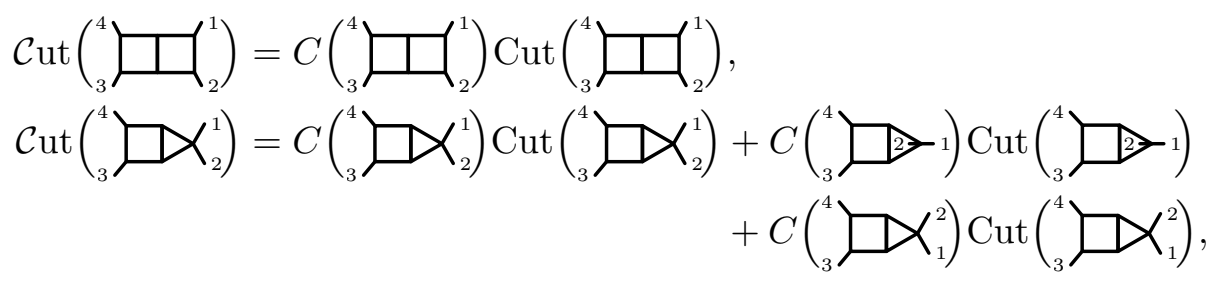

where the $C(\ldots)$ factors are constructed from the colour vertices $(2.2)$ understood in the sense of figure 1. Notably, the two cuts are expanded onto different bases of colour structures, which obscure the irreducible numerator projection. However, taking a factorisation limit of eq. (2.16) we can obtain an alternative decomposition of the parent cut that is more useful for the subtraction:

$$
\mathcal{C u t}\left({ }_{3}^{4} \square_{2}\right)=C\left({ }_{3}^{4} \square \times_{2}^{1}\right) \operatorname{Cut}\left({ }_{3}^{4} \square_{2}^{1}\right)+C\left({ }_{3}^{4} \square_{1}\right) \operatorname{Cut}\left({ }_{3}^{4} \square_{2} V^{1}\right) .
$$

Clearly, the parent irreducible numerator obtained from this colour expansion facilitates the colour-ordered projection (2.14) applied to the child $\operatorname{Cut}(\mathcal{D})$. Furthermore, here the second ordered numerator of eq. (2.17) is different from the one in eq. (2.15) only by a sign. More generally, the irreducible numerators mandated by alternative parent decompositions should be obtained using KK relations [64] from the set of irreducible numerators participating in the original parent colour decomposition, as we shall explain in more detail in section 3.

This example also shows that the reasoning behind the colour-ordered projection (2.14) of the hierarchy subtraction (2.11) is basically equivalent to that of the tree-level colour ordering (2.1), which is behind the off-shell [68] and on-shell [69-71] recursion for tree-level 


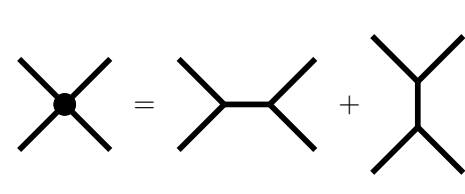

(a)

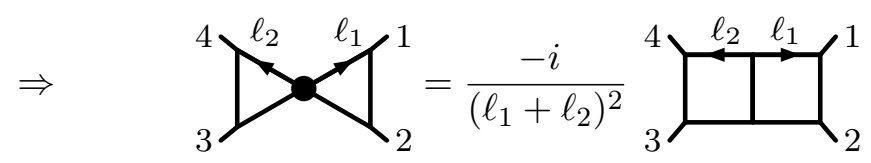

(b)

Figure 2. The quartic ordered subtraction vertex contains a maximum of two subgraphs (a). Inside the two-loop butterfly topology (b), only the second subgraph's propagator is loop-dependent and contributes to the hierarchy subtraction.

objects. Indeed, any $\Delta_{j}$ generated by eq. (2.14) belongs to some higher-level unordered numerator $\tilde{\Delta}_{\mathcal{J}}$, inside of which its associated colour factor is $T_{j}=\prod_{k} T\left(\sigma_{k}\right)$ (with repeating colour indices summed over), just in the same way as a tree amplitude contribution with one or more specified propagators can be related to a product of lower-point amplitudes with similarly factorised colour factors. Then due to the $\mathrm{SU}\left(N_{c}\right)$-completeness relation

$$
T_{i \bar{\jmath}}^{a} T_{k \bar{l}}^{a}=\delta_{i \bar{l}} \delta_{k \bar{\jmath}}-\frac{1}{N_{c}} \delta_{i \bar{\jmath}} \delta_{k \bar{l}}
$$

products of trace-based colour factors can be mapped to higher-point colour factors. For instance, in the factorisation limit of an $n$-point vertex into $(k+1)$ - and $(n-k+1)$-point ones, the product of two trace-based colour vertices is

$$
\begin{aligned}
T(1,2, \ldots, k, l) T(l, k+1, \ldots, n)= & T(1,2, \ldots, k, k+1, \ldots, n) \\
& +(-1)^{k+1} T(k, \ldots, 2,1, k+1, \ldots, n) \\
& -\frac{1}{N_{c}} \widetilde{T}(1,2, \ldots, k) \widetilde{T}(k+1, \ldots, n),
\end{aligned}
$$

where the $1 / N_{c}$ term involves $\widetilde{T}(1, \ldots, k) \equiv \operatorname{Tr}\left(T^{a_{1}} \ldots T^{a_{k}}\right)+(-1)^{k+1} \operatorname{Tr}\left(T^{a_{k}} \ldots T^{a_{1}}\right)$, differing from eq. (2.2) by the sign of the second term. The terms with $\widetilde{T}(\ldots)$ are irrelevant for the current discussion, since they cancel altogether in the full-colour integrand due to photon-decoupling identities among $\Delta_{i}$, the validity of which will be shown in section 3.1. The first two terms in eq. (2.19), however, are colour factors of $n$-point vertices, which thus produce poles in lower-level colour-ordered cuts that need to be removed.

The resulting ordered hierarchy subtraction is by now standard in multi-loop calculations at leading colour (see e.g. ref. [72] for exposition). Higher $n$-point vertices can in principle generate subgraphs with up to $(n-3)$ new propagators, as shown in figures 2 a and 3 (where a dotted higher-point vertex denotes the subtraction contributions associated to that vertex). However, since the raison d'être of these subgraphs is to remove poles from a given colour-ordered cut, the resulting topologies that do not generate additional loop-dependent propagators do not correspond to higher-level poles. For example, every ordered quartic vertex can give rise to two subgraphs shown in figure $2 \mathrm{a}$, but in application to a two-loop numerator $\Delta(\searrow)$ their denominators are $s_{12}$ and $\left(\ell_{1}+\ell_{2}\right)^{2}$, so only the second subgraph actually contributes to the hierarchy subtraction, as depicted in figure $2 \mathrm{~b}$. Moreover, the number of propagators in the resulting loop graphs must not 


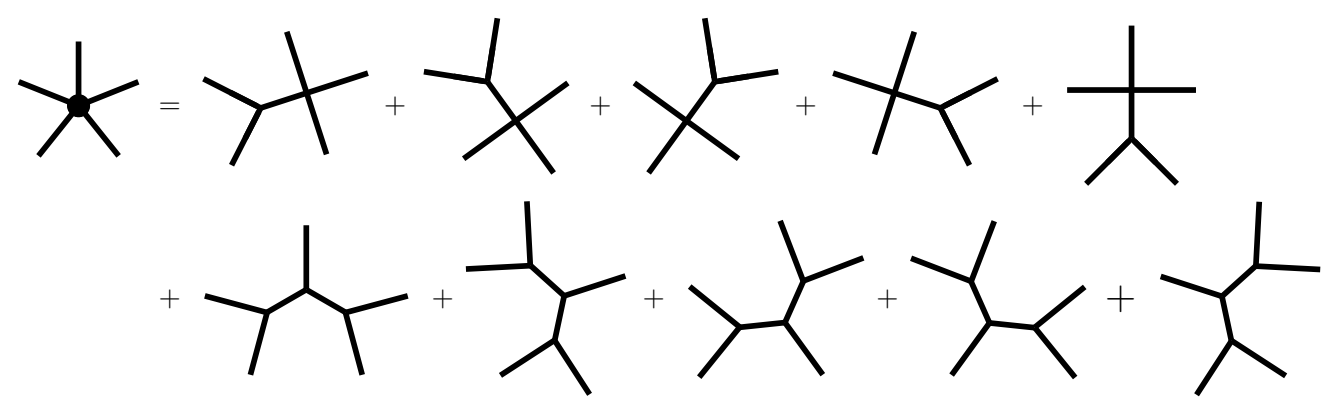

Figure 3. The quintic ordered subtraction vertex contains a maximum of five one-propagator and five two-propagator subgraphs. These subgraphs are not Feynman diagrams of Yang-Mills theory; for instance, the six-point subtraction vertex contains even subgraphs involving five-point vertices.

exceed $L(L+9) / 2$, as that would correspond to an overconstrained beyond-maximal cut with non-existent pole.

To summarise, eq. (2.14) defines irreducible numerators $\Delta_{i}$ from colour-ordered cuts such that they each carry a new piece of the kinematic information invisible to the preceding more constrained cuts. The loop amplitude (2.12) can then be consistently rewritten as a sum over ordered one-particle irreducible graphs: ${ }^{4}$

$$
\mathcal{A}_{n}^{(L)}=g^{n+2 L-2} \sum_{i \in 1 \text { PI graphs }} \int \frac{\mathrm{d}^{L D} \ell}{(2 \pi)^{L D}} \frac{T_{i} \Delta_{i}}{S_{\mathcal{I}} \prod_{l \in i}\left(-i D_{l}\right)} .
$$

Note that since the trace-based colour factors $T_{i}$ are linearly dependent, this is not a decomposition into a colour basis. Rather it is an expression given in terms of colour factors associated with loop-singularity kinematic structures, defined in terms of colourordered objects.

\subsection{Four-point two-loop example}

As a simple nontrivial example of the trace-based colour decomposition (2.20), let us consider the two-loop Yang-Mills amplitude with four plus-helicity gluons, first computed in ref. [73]. Its helicity configuration is fully symmetric, and its tree-level counterpart vanishes, which is known to result in a much simpler structure than in a generic four-point two-loop amplitude $[4,74]$. In fact its only nonzero irreducible numerators are produced by two maximal and one next-to-maximal coloured cuts, i.e.

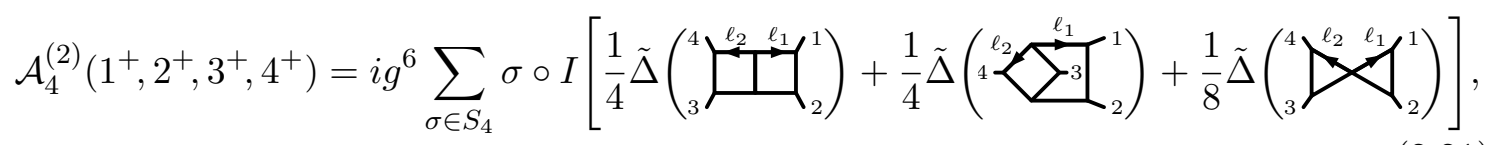

where the factors of $1 / 4$ and $1 / 8$ are there to remove overcounting of equivalent topologies in the permutation sum $S_{4}$. Here and below we use a shorthand notation for the combination

\footnotetext{
${ }^{4}$ In the following sections, the factors of $i$, inherited by eqs. (2.12) and (2.20) from the cut convention $i / D_{l} \rightarrow \delta\left(D_{l}\right)$, will be reabsorbed into $\Delta_{i}$ so as to produce an overall factor of $i^{L-1}$.
} 
of the appropriate integration measure and denominators:

$$
I\left[\tilde{\Delta}_{\mathcal{I}}\right] \equiv \int \frac{\mathrm{d}^{D} \ell_{1} \ldots \mathrm{d}^{D} \ell_{L(\mathcal{I})}}{(2 \pi)^{D L(\mathcal{I})}} \frac{\tilde{\Delta}_{\mathcal{I}}}{\prod_{l \in \mathcal{I}} D_{l}}
$$

The coloured irreducible numerators for the maximal topologies are extremely simple and depend solely on the higher-dimensional components of loop momenta,

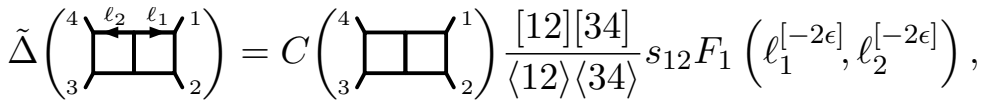

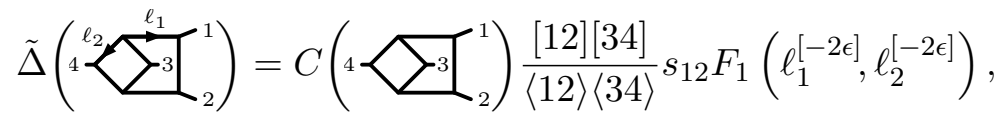

through the function

$$
F_{1}\left(\ell_{1}^{[-2 \epsilon]}, \ell_{2}^{[-2 \epsilon]}\right)=\left(D_{s}-2\right)\left(\mu_{11} \mu_{22}+\left(\mu_{11}+\mu_{22}\right)\left(\mu_{11}+\mu_{22}+2 \mu_{12}\right)\right)+16\left(\mu_{12}^{2}-\mu_{11} \mu_{22}\right)
$$

written in terms of scalar products $\mu_{i j} \equiv \ell_{i}^{[-2 \epsilon]} \cdot \ell_{j}^{[-2 \epsilon]}$, and the spin dimension $D_{s}$ that effectively interpolates between the 't Hooft-Veltman regularisation scheme [75], $D_{s}=4-2 \epsilon$, and the four-dimensional helicity scheme [76], $D_{s}=4$.

The coloured numerator for the next-to-maximal topology contains a four-point vertex with three possible colour orderings,

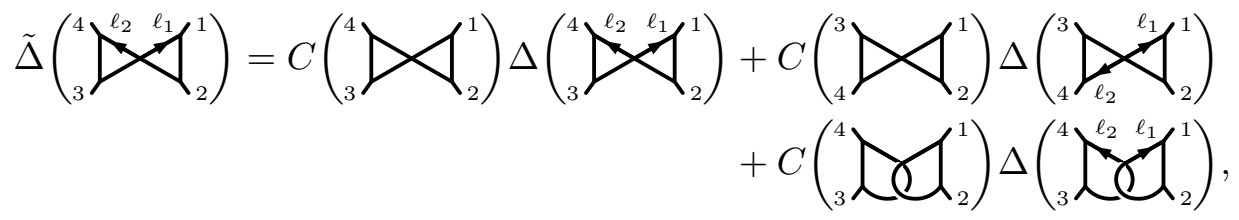

where the colour factors $C_{i}$ are obtained by plugging in the trace-based vertices (2.2),

$$
\begin{aligned}
& C\left(\left.{ }_{3}^{4}\right|_{2} ^{1}\right)=T(1, e, a) T(2, b, e) T(3, f, c) T(4, d, f) T(a, b, c, d), \\
& C\left(\left.{ }_{4}^{3}\right|_{2} ^{1}\right)=T(1, e, a) T(2, b, e) T(3, f, c) T(4, d, f) T(a, b, d, c), \\
& C\left({ }_{3}^{4} \mathrm{Cl}_{2}^{1}\right)=T(1, e, a) T(2, b, e) T(3, f, c) T(4, d, f) T(a, c, b, d) .
\end{aligned}
$$

Whilst we now have the full decomposition in the trace basis, this expression can be further simplified. To demonstrate this, consider the form of the ordered numerators $\Delta_{i}[53,73]$ :

$\left.\Delta\left({ }_{3}^{4}\right\rangle^{\ell_{2}} \ell_{1}^{\ell_{1}}{ }^{1}\right)=\frac{[12][34]}{\langle 12\rangle\langle 34\rangle}\left\{4\left(D_{s}-2\right)\left(\mu_{11}+\mu_{22}\right) \mu_{12}+\left(D_{s}-2\right)^{2} \mu_{11} \mu_{22} \frac{\left(\ell_{1}+\ell_{2}\right)^{2}+s_{12}}{s_{12}}\right\}$,

$\Delta\left(\left.{ }_{4}^{3} D_{\ell_{2}}^{\ell_{1}}\right|_{2} ^{1}\right)=\frac{[12][34]}{\langle 12\rangle\langle 34\rangle}\left\{-4\left(D_{s}-2\right)\left(\mu_{11}+\mu_{22}\right) \mu_{12}+\left(D_{s}-2\right)^{2} \mu_{11} \mu_{22} \frac{\left(\ell_{1}-\ell_{2}-p_{12}\right)^{2}+s_{12}}{s_{12}}\right\}$,

$\Delta\left(\left.{ }_{3}^{4} \mathrm{~L}^{\ell_{2}} \mathrm{\ell}_{2}^{1}\right|_{2} ^{1}\right)=-\frac{[12][34]}{\langle 12\rangle\langle 34\rangle}\left(D_{s}-2\right)^{2} \mu_{11} \mu_{22} \frac{\left(\ell_{1}+\ell_{2}\right)^{2}+\left(\ell_{1}-\ell_{2}-p_{12}\right)^{2}+2 s_{12}}{s_{12}}$. 
We can immediately observe that they obey

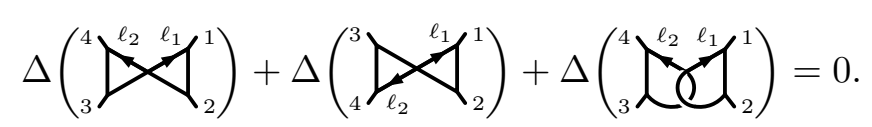

We dub this a numerator KK relation in that it is inherited from the same relation between the colour-ordered cuts. To understand how this arises note that the three corresponding cuts contain identical three-point amplitudes and differ only by the orderings of the fourpoint amplitude in the middle, which naturally obey the KK relation [64]

$$
A\left(\ell_{1}, \ell_{2}, \ell_{3}, \ell_{4}\right)+A\left(\ell_{1}, \ell_{3}, \ell_{2}, \ell_{4}\right)+A\left(\ell_{1}, \ell_{2}, \ell_{4}, \ell_{3}\right)=0
$$

where $\ell_{3}=-\ell_{2}-p_{12}, \ell_{4}=-\ell_{1}+p_{12}$. Therefore,

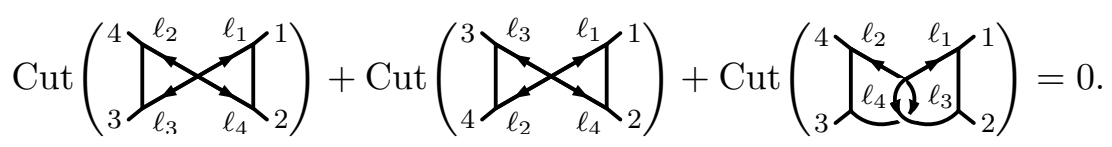

Each of these cuts is given by its irreducible numerator and the higher-level numerator from the maximal topology, as indicated in figure 2, but the latter cancel in the sum:

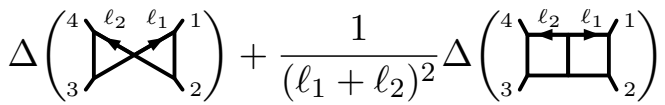

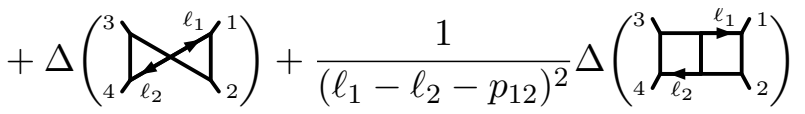

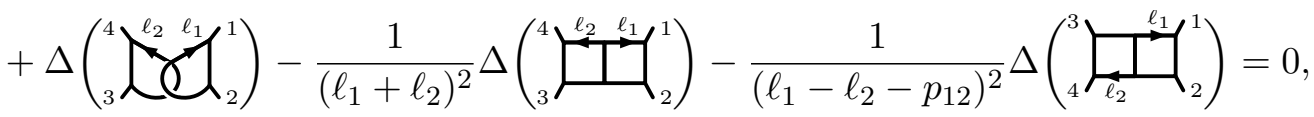

yielding exactly eq. (2.28) on the cut.

To ensure that it holds away from the cut kinematics, we rely on the bijection properties of the cut-numerator map (2.7). In other words, the loop variables chosen for the three orderings $(2.27 \mathrm{a}),(2.27 \mathrm{~b})$ and $(2.27 \mathrm{c})$ should be consistent with a single choice of the irreducible monomials for the butterfly topology in the unordered sense. At first glance, it may seem not to be the case, as on the cut kinematics among the three appearing variables

$$
s_{12}, \quad\left(\ell_{1}+\ell_{2}\right)^{2}=2 \ell_{1} \cdot \ell_{2}, \quad\left(\ell_{1}-\ell_{2}-p_{12}\right)^{2}=-2 \ell_{1} \cdot \ell_{2}-s_{12},
$$

only any two can be considered as independent monomials (along with the factor $\mu_{11} \mu_{22}$ ). However, there is only two unique combinations that appear in the numerators eq. (2.27):

$$
\left(\ell_{1}+\ell_{2}\right)^{2}+s_{12}=2 \ell_{1} \cdot \ell_{2}+s_{12}, \quad\left(\ell_{1}-\ell_{2}-p_{12}\right)^{2}+s_{12}=-2 \ell_{1} \cdot \ell_{2},
$$

that do form an independent monomial set, which is equivalent to $\left\{\mu_{11} \mu_{22}, \mu_{11} \mu_{22}\left(\ell_{1} \cdot \ell_{2}\right)\right\}$. The choice of the set (2.33) is dictated by the empirical knowledge that the inclusion of harmless $\ell_{1}^{2}, \ell_{2}^{2}$, etc., inside the monomials results in vanishing lower-level numerators. 
In section 3.1 we will give a more general argument for the validity of the KK relations [64] between irreducible numerators. Now let us proceed with taking advantage of eq. (2.28). Plugging it in the coloured numerator (2.25), we obtain

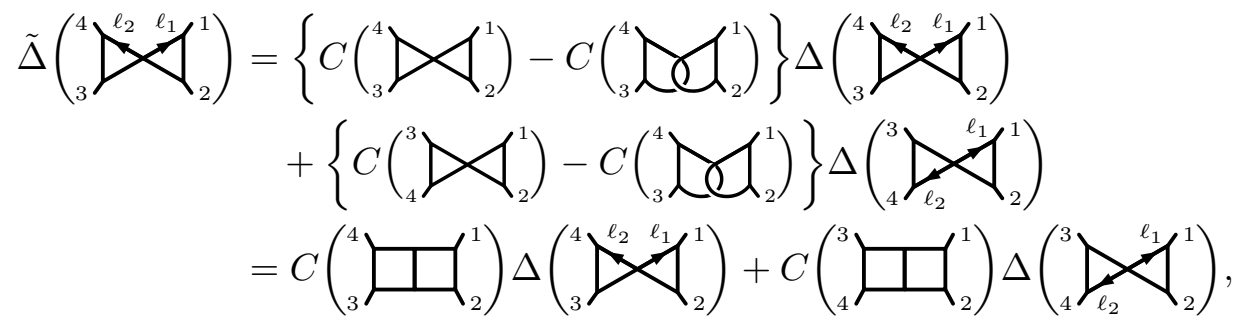

where in the last line we used that the colour traces in eq. (2.26) satisfy

$$
T(a, b, c, d)-T(a, c, b, d)=\tilde{f}^{b c e} \tilde{f}^{e d a}, \quad T(a, b, d, c)-T(a, c, b, d)=\tilde{f}^{b d e} \tilde{f}^{e c a},
$$

i.e. we reversed the familiar colour reduction for the four-point cubic diagrams. Comparing eqs. (2.25) and (2.34), note that the two colour decompositions of the butterfly numerator are obtained respectively from the two expressions for the four-point tree,

$$
\begin{aligned}
\mathcal{A}_{4}^{(0)} & =T(1,2,3,4) A(1,2,3,4)+T(1,2,4,3) A(1,2,4,3)+T(1,3,2,4) A(1,3,2,4) \\
& =\tilde{f}^{23 e} \tilde{f}^{e 41} A(1,2,3,4)+\tilde{f}^{24 e} \tilde{f}^{e 31} A(1,2,4,3),
\end{aligned}
$$

the latter being the DDM decomposition [66].

Since we have thus eliminated the colour-ordered butterfly numerator $(2.27 \mathrm{c})$, and the numerators $(2.27 \mathrm{a})$ and $(2.27 \mathrm{~b})$ are the same up to relabelling, we can rewrite the full amplitude (2.21) in a compact form:

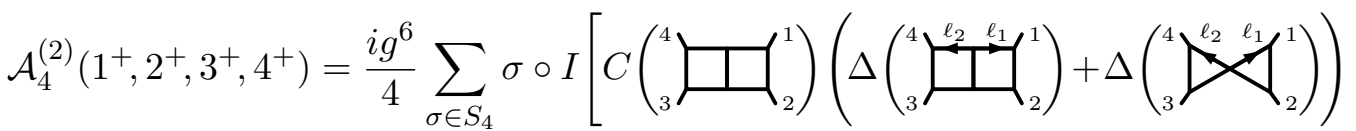

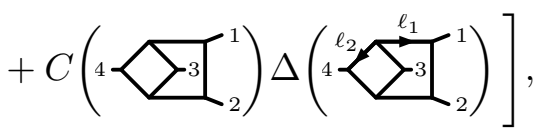

where the colour factor of the double-box irreducible numerator (2.23a) is now explicit and shared with the butterfly contribution. Apart from being more concise, interestingly, this representation coincides with the one obtained in the original calculation [73].

\section{DDM-based colour decomposition}

The lesson that we can learn from the example in section 2.3 is that the trace-based colour decomposition (2.20) runs over an overcomplete set of ordered cut topologies, in the same way as the trace decomposition of the tree amplitude (2.1) involves amplitudes that are linearly dependent under KK relations [64]:

$$
A(1, \alpha, n, \beta)=(-1)^{|\beta|} \sum_{\sigma \in \alpha \amalg \beta^{T}} A(1, \sigma, n) .
$$




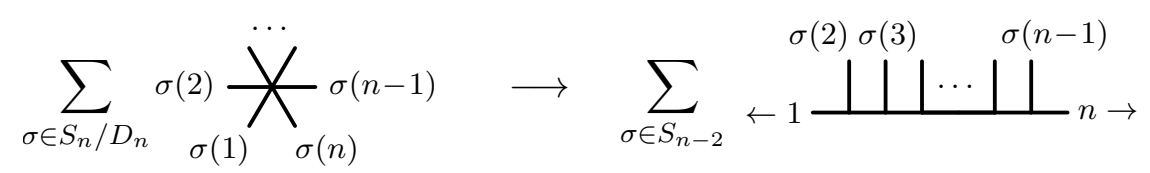

Figure 4. Graphic representation of the process of obtaining the DDM colour factors from traces via "stretching" of the vertex by any two edges (here chosen to be 1 and $n$ ).

These relations resolve any colour-ordered amplitudes in terms of the subset of $(n-2)$ ! amplitudes with two particles fixed in colour-adjacent positions. If one plugs this solution back to into the trace decomposition (2.1), the resulting colour coefficients turn out to combine into single strings of structure constants:

$$
\begin{aligned}
& \mathcal{A}_{n}^{(0)}=g^{n-2} \sum_{\sigma \in S_{n-2}} \tilde{f}^{a_{1} a_{\sigma(2)} b_{1}} \tilde{f}^{b_{1} a_{\sigma(3)} b_{2}} \ldots \tilde{f}^{b_{n-4} a_{\sigma(n-2)} b_{n-3}} \tilde{f}^{b_{n-3} a_{\sigma(n-1)} a_{n}} \\
& \times A(1, \sigma(2), \ldots, \sigma(n-1), n)
\end{aligned}
$$

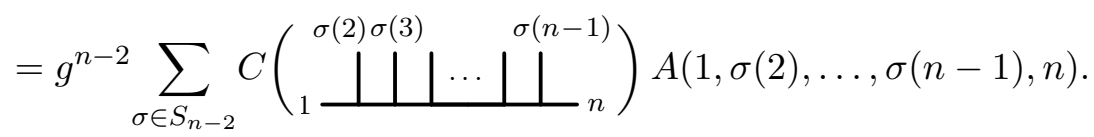

This is the DDM colour decomposition [65, 66], and with respect to eq. (2.1) it avoids $(n-2) !(n-3) / 2$ colour-ordered amplitudes. Its four-point version, eq. (2.36), when applied inside the two-loop butterfly numerator (2.34), relates the two decompositions of section 2.3.

More generally, once the KK relations for irreducible numerators are taken into account and their coefficients combined into the DDM-based colour factors, we can rewrite the trace-based decomposition $(2.20)$ as

$$
\mathcal{A}_{n}^{(L)}=i^{L-1} g^{n+2 L-2} \sum_{i \in \text { KK-independent 1PI graphs }} \int \frac{\mathrm{d}^{L D} \ell}{(2 \pi)^{L D}} \frac{C_{i} \Delta_{i}}{S_{\mathcal{I}} \prod_{l \in i} D_{l}},
$$

where we have also reabsorbed factors of $i$ into the numerators to produce the overall factor of $i^{L-1}$. The colour factors $C_{i}$ will be naturally given by trivalent diagrams, but their concrete forms depend on the way the KK reduction is realised, i.e. on the choice of the KK-independent subset of ordered 1PI graphs. The process of elimination the redundant topologies involves picking two edges of a higher-point vertex and "stretching" it by these edges to produce a sum over DDM "half-ladder" colour factors, as illustrated by figure 4, each multiplied by its corresponding permutation of the original vertex. The resulting subset of ordered topologies thus depends on the choice of the "stretched" edges.

In the following sections, we shall demonstrate how to achieve this automatically by inserting the DDM tree decomposition rather than the trace basis decomposition. But first, let us generalise the reasoning of section 2.3 on the validity of the KK relations for irreducible numerators.

\subsection{Kleiss-Kuijf relations for irreducible numerators}

In this section we argue that irreducible numerators can be chosen so as to inherit the same KK relations from the colour-ordered unitarity cuts, from which they are derived. 
In the top-down approach, the definition of an irreducible numerator involves subtraction of the higher-level numerators from its cut, as summarised in eq. (2.14). Let us rewrite it with explicit reference to the level in the graph hierarchy, labelled by $l, l+1, \ldots, l_{\max }$ :

$$
\Delta_{i}^{(l)}=\mathrm{Cut}_{i}^{(l)}-\sum_{\operatorname{uncut} D_{i j}} \frac{\Delta_{i j}^{(l+1)}}{D_{i j}}-\ldots-\sum_{\operatorname{uncut} D_{i j} \ldots D_{i k}} \frac{\Delta_{i j \ldots k}^{\left(l_{\max }\right)}}{D_{i j} \ldots D_{i k}} .
$$

Here every $\Delta_{i j \ldots k}^{\left(l^{\prime}\right)}$ corresponds to a cut obtained from $\mathrm{Cut}_{i}^{(l)}$ by constraining additional loop propagators $D_{i j}, \ldots, D_{i k}$; it is subtracted from $\mathrm{Cut}_{i}^{(l)}$ to ensure polynomial nature of $\Delta_{i}^{(l)}$. Obviously, the top-level numerators $\Delta_{i}^{\left(l_{\max }\right)}$ obey the KK relations of $\mathrm{Cut}_{i}^{\left(l_{\max }\right)}$ on their cut kinematics, as they correspond to maximal cuts without any subtractions. In order for this to be true for general (off-shell) kinematics, we choose the same basis of numerator functions for each term in a KK relation, effectively associating the basis with the cut condition. In this way, the KK relation of the irreducible numerators follows from that of the cuts. Having established the validity of the KK relations for top-level numerators, we shall now construct a recursive argument for the lower levels.

Our argument will rely heavily on the observation $[66,77]$ that the KK relations can be viewed as a consequence of the antisymmetry of a cubic expansion for colour-ordered amplitudes. Indeed, since colour content of a gauge theory consists only of trivalent structures $\tilde{f}^{a b c}$ and $T_{i \bar{\jmath}}^{a}$, any Feynman diagram with insertions of higher-point Feynman vertices can be absorbed into purely cubic diagrams, modifying their kinematic (reducible) numerators $n_{i}$ but leaving any gauge-invariant object unchanged. This ensures the existence of a cubic expansion for any tree or loop amplitude. The antisymmetry under a trivalent-vertex flip

$$
C_{i}=-C_{j} \quad \Leftrightarrow \quad n_{i}=-n_{j}
$$

in this context simply means that $i$ and $j$ are essentially one cubic graph. At tree level this means that, after reduction to colour traces, different colour-ordered amplitudes pick up the same cubic graph with different signs. The KK relations, being purely colour-algebra statements without any reference to kinematics, hold simply due to cancellation among the kinematic parts of the same cubic graphs occurring twice with opposite signs [66, 77].

Now consider a linear combination of cuts that vanishes due to a KK relation:

$$
\begin{aligned}
0=\sum_{\sigma_{j}} A\left(\sigma_{j}\right) \propto \sum_{i} \mathrm{Cut}_{i}^{(l)}=\sum_{i} \Delta_{i}^{(l)} & +\sum_{i} \sum_{\text {uncut } D_{i j}} \frac{\Delta_{i j}^{(l+1)}}{D_{i j}}+\ldots \\
& +\sum_{i} \sum_{\text {uncut } D_{i j} \ldots D_{i k}} \frac{\Delta_{i j \ldots k}^{\left(l_{\max }\right)}}{D_{i j} \ldots D_{i k}} .
\end{aligned}
$$

This means that the cuts $\mathrm{Cut}_{i}^{(l)}$ contain a common, up to permutations, vertex $j$ such that the corresponding colour-ordered tree amplitudes $A\left(\sigma_{j}\right)$ produce pairwise annihilating cubic diagrams. Supposing that all the KK relations for the higher-level numerators are already proven, we can assume that for all such irreducible numerators $\Delta_{i j \ldots k}^{\left(l^{\prime}>l\right)}$ on the right-hand side there exist an expansion in purely cubic graphs. We will not need any specific form of 
such kinematic diagrams; all we are going to require is their existence. These cubic graphs must contain subgraphs that correspond to permutations of the same vertex $j$. Indeed, the subtraction procedure that generated those higher levels in the first place started with the cut graphs containing that vertex and then proceeded by exposing loop-dependent propagators inside of it and other vertices. Therefore, when each of the generated higherlevel graphs is expanded into purely cubic graphs, they will all contain the propagators of the original cut and hence the subgraphs corresponding to the vertex $j$. From now on, we can concentrate on what is happening inside these subgraphs.

Consider the cubic subgraphs generated by two numerators of adjacent levels $l^{\prime}$ and $l^{\prime}+1$. Since the latter's graph contains one more exposed propagator than the former's from the start, this propagator will be present in all of its cubic graphs, thus picking a subset of the cubic graphs of the former. Hence each cubic subgraph produced by any higher-level numerator is isomorphic to some cubic subgraph of the vertex $j$. But the permutation sum on the left-hand side guaranteed that every cubic subgraph will appear in expansion of two (or an even number of) colour-ordered amplitudes with opposite signs. Since the subtraction procedure was only sensitive to the propagator structure, any cubic subgraph produced by some higher-level numerator must be accompanied by a twin with the opposite orientation, produced by another higher-level numerator. In other words, the fact that the higher-level numerators $\Delta_{i j \ldots k}^{\left(l^{\prime}>l\right)}$ do not contain all possible cubic graphs cannot interfere with the cancellations coming from the overall permutation sum. Therefore, we conclude that the cubic diagrams must cancel at all higher levels, and then the equality (3.6) itself proves the KK relation for the level at hand, as needed for our inductive argument.

More formally, if $\mathcal{C} \equiv\left\{\Gamma_{j} \in \sum_{i} \mathrm{Cut}_{i}^{(l)}\right\}$ is the set of all oriented cubic graphs on the left-hand side of eq. (3.6), then it can be divided into two equal sets $\mathcal{C}^{+} \cup \mathcal{C}^{-}=\mathcal{C}$ that differ only by orientation. For each level $l^{\prime}>l$ on the right-hand side, there is a map of the numerator graphs $\Gamma_{j}^{\left(l^{\prime}\right)}$ to a subset of $\mathcal{C}$, representing the trivalent expansion of the higher-level numerators. By construction, since it only involves exposing loop-dependent propagators, this map is symmetric with respect to both orientations $\mathcal{C}^{+}$and $\mathcal{C}^{-}$. Therefore, for $\forall \Gamma_{j}^{\left(l^{\prime}\right)} \in \sum_{i ; j, \ldots, k} \Delta_{i j \ldots k}^{\left(l^{\prime}\right)} /\left(D_{i j} \ldots D_{i k}\right)$, there $\exists\left(-\Gamma_{j}^{\left(l^{\prime}\right)}\right) \in \sum_{i ; j, \ldots, k} \Delta_{i j \ldots k}^{\left(l^{\prime}\right)} /\left(D_{i j} \ldots D_{i k}\right)$ with an opposite orientation. This guarantees cancellation of the cubic diagrams from all levels, except $l$, and the equality (3.6) itself then proves the KK relation for $\Delta_{i}^{(l)}$, closing the induction.

\subsection{One-loop case}

In this section we consider the application of the DDM-based colour decomposition (3.3) to a purely gluonic one-loop amplitude. As one would expect, we recover the one-loop DDM colour decomposition [66]:

$$
\begin{aligned}
\mathcal{A}_{n}^{(1)} & =g^{n} \sum_{\sigma \in S_{n} / D_{n}} \tilde{f}^{b_{1} a_{\sigma(1)} b_{2}} \tilde{f}^{b_{2} a_{\sigma(2)} b_{3}} \ldots \tilde{f}^{b_{n} a_{\sigma(n)} b_{1}} A^{(1)}(\sigma(1), \sigma(2), \ldots, \sigma(n)) \\
& =g^{n} \sum_{\sigma \in S_{n} / D_{n}} C(\underbrace{}_{\sigma(2)} \overbrace{\sigma(n)} \sigma(n-1)) A^{(1)}(\sigma(1), \sigma(2), \ldots, \sigma(n)),
\end{aligned}
$$




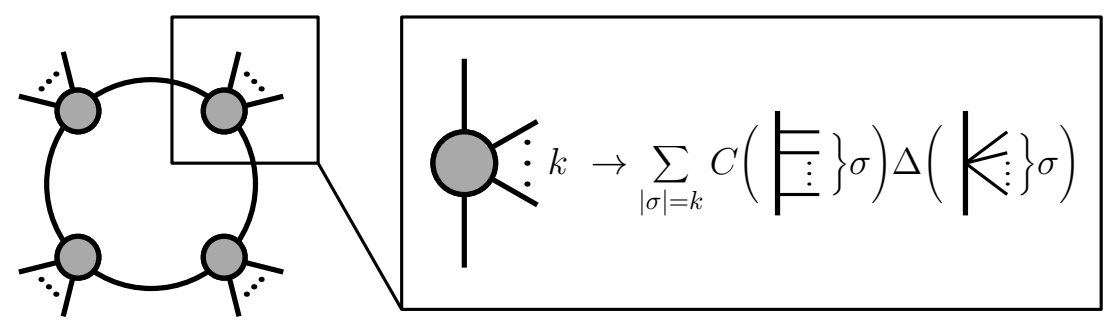

Figure 5. Inserting the DDM tree basis into coloured cuts of a one-loop amplitude. As shown in the inset, "stretching" by the two internal edges gives a sum over permutations of the external legs.

where $A^{(1)}$ are leading-colour partial amplitudes. Recall that these objects can be defined at any loop order as gauge-invariant coefficients of the leading single-trace colour factors:

$$
\begin{aligned}
\mathcal{A}_{n}^{(L)}=i^{L-1} g^{n+2 L-2}\{ & N_{c}^{L} \sum_{\sigma \in S_{n} / Z_{n}} \operatorname{Tr}\left(T^{a_{\sigma(1)}} T^{a_{\sigma(2)}} \ldots T^{a_{\sigma(n)}}\right) A^{(L)}(\sigma(1), \sigma(2), \ldots, \sigma(n)) \\
& \left.+\mathcal{O}\left(N_{c}^{L-1}\right)\right\} .
\end{aligned}
$$

At one loop, all the subleading partial amplitudes are known to be linear combinations of the leading ones [78], and thus the full amplitude can be written in terms of $A^{(1)}$ only, as implemented by the one-loop DDM decomposition (3.7).

To see how eq. (3.7) follows from eq. (3.3), let us consider a generic one-loop topology depicted in figure 5 . In $D=4-2 \epsilon$ dimensions, it consists of up to five vertices connected into a loop, each with 3 or more edges, two of which are internal. To use our DDMbased prescription, it is natural to use these internal edges as the "stretching" ends for the DDM colour factors, so that the resulting ordered graphs are enumerated by permutations of external legs on one side of the resulting "half-ladder". This procedure eliminates ordered topologies with some of the external legs sticking inside the loop; they correspond to valid colour-ordered cuts but are redundant under the KK relations. Therefore, the remaining ordered topologies are pentagons, boxes, triangles, bubbles and tadpoles with all external particles looking, for definiteness, outside the loop. Moreover, all colour factors will be loops of structure constants, as in eq. (3.7), differing only by permutations of external legs. The ordered topologies that will contribute to the kinematic coefficient of $\tilde{f}^{b_{1} a_{1} b_{2}} \tilde{f}^{b_{2} a_{2} b_{3}} \ldots \tilde{f}^{b_{n} a_{n} b_{1}}$, for instance, will be precisely the colour-ordered amplitude ${ }^{5}$

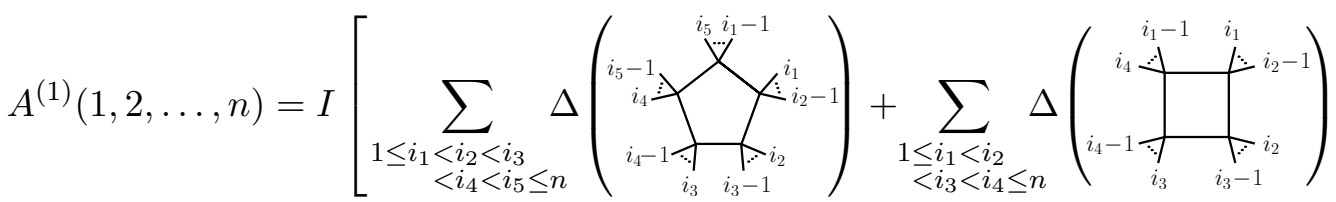

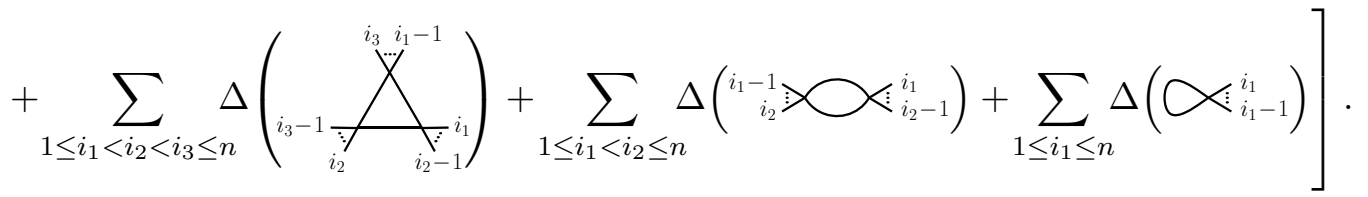

\footnotetext{
${ }^{5}$ For generality, we allowed tadpoles and bubbles on external legs in the one-loop adjoint-representation amplitude (3.9), even though in dimensional regularisation they integrate to zero in the massless case.
} 
The only subtlety here is that fixing the two internal edges inside the bubble topologies results in pairs of equivalent ordered topologies. For example, the two DDM decompositions inside the coloured bubble $(1,2,3 ; 4,5)$ produce the orderings $(1,2,3 ; 4,5)$ and $(3,2,1 ; 5,4)$ as distinct permutations, though obviously with equivalent colour factors $\pm \tilde{f}^{b_{1} a_{1} b_{2}} \tilde{f}^{b_{2} a_{2} b_{3}} \tilde{f}^{b_{3} a_{3} b_{4}} \tilde{f}^{b_{4} a_{4} b_{5}} \tilde{f}^{b_{5} a_{5} b_{1}}$. This precisely cancels the bubble symmetry factor $S_{\text {bub }}=2$, thereby absent from the leading-colour amplitude (3.9). A similar argument takes care of the tadpole symmetry factor $S_{\mathrm{tad}}=2$. This concludes the check that at one loop our DDM-induced multi-loop decomposition (3.3) reduces to the proper DDM decomposition (3.7).

\subsection{Two-loop case}

Let us now consider the case of the two-loop colour decomposition by cuts [54]. At two loops, there are two basic topologies: the "pure two-loop" topology and the "butterfly", given in figures 6 and 7, respectively. In order to find a KK-independent basis of colourordered objects, we wish once more to insert the DDM decomposition into the coloured cuts. As at one loop we must make choices of which two gluons to hold fixed in the DDM tree. Here the procedure is more complicated as some coloured trees contain more than two loop-dependent legs. We find trees with two and three loop-dependent legs in the pure two-loop topologies and two and four loop-dependent legs in the butterfly topologies. Let us consider these cases in turn.

- Two loop legs: as at one loop, perform the DDM decomposition holding the two loop edges fixed and permuting over the external legs. See inset (a) of figure 6.

- Three loop legs: choose two loop edges to fix and DDM-decompose, permuting across the external legs and the third loop edge. See inset (b) of figure 6 .

- Four loop legs: choose one side on which to fix two of the loop edges and permute over the external legs and the two loop edges on the other side. See figure 7.

The canonical choices made at two loops remove some of the symmetry properties of the coloured diagrams in order to ensure KK independence. Note that there is a degree of arbitrarity in this procedure, which was not present at one loop, where every tree in a cut has exactly two loop-dependent legs.

It should also be noted that a KK-independent numerator basis at two loops does not enjoy the complete consistency between levels which is found at one loop. Specifically, when performing the hierarchy subtraction of a lower-level topology, one may run into an ancestor graph that was not in the KK-independent basis defined by the DDM-based colour decomposition of the coloured ancestor numerator. However, as the terms in that decomposition form a KK-independent basis, one can always use the KK relations to obtain the colour-ordered ancestor, needed for lower-level subtraction. For example, if the canonical procedure in figure $6 \mathrm{~b}$ colour-decomposes the following double-triangle topology as

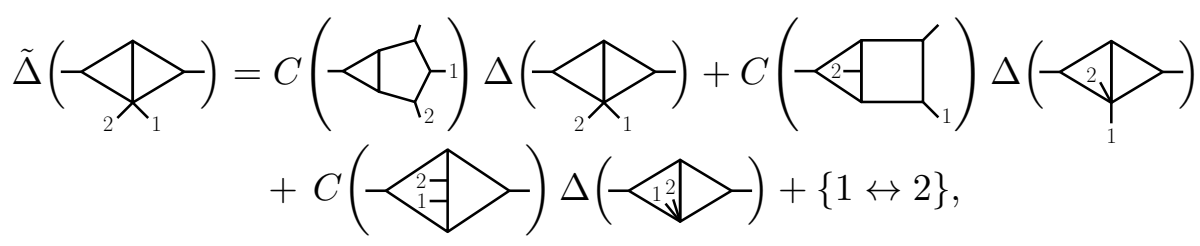




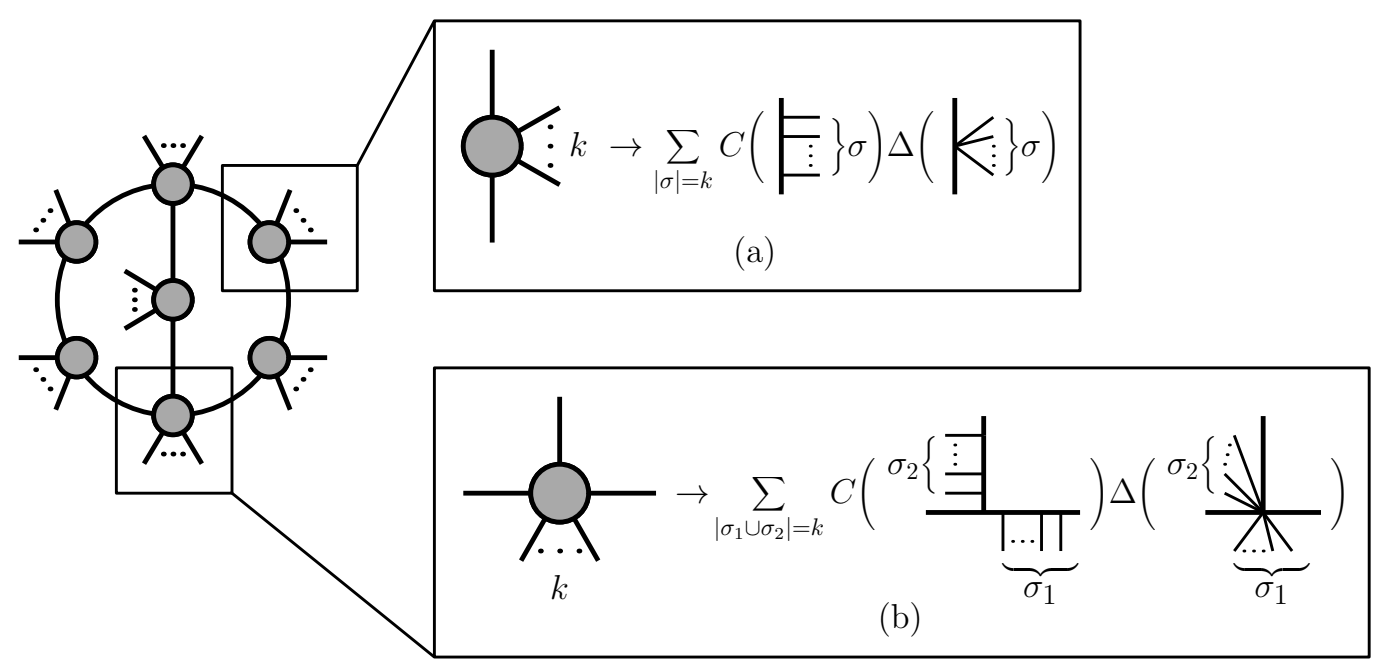

Figure 6. Inserting the DDM tree basis into a pure two-loop coloured cut. The upper inset (a) shows the case of two loop-dependent legs (thus equivalent to the one-loop decomposition in figure 5), while the lower inset (b) shows the case of three loop-dependent legs. The sums run over the permutations of the external legs in the tree-level amplitude.

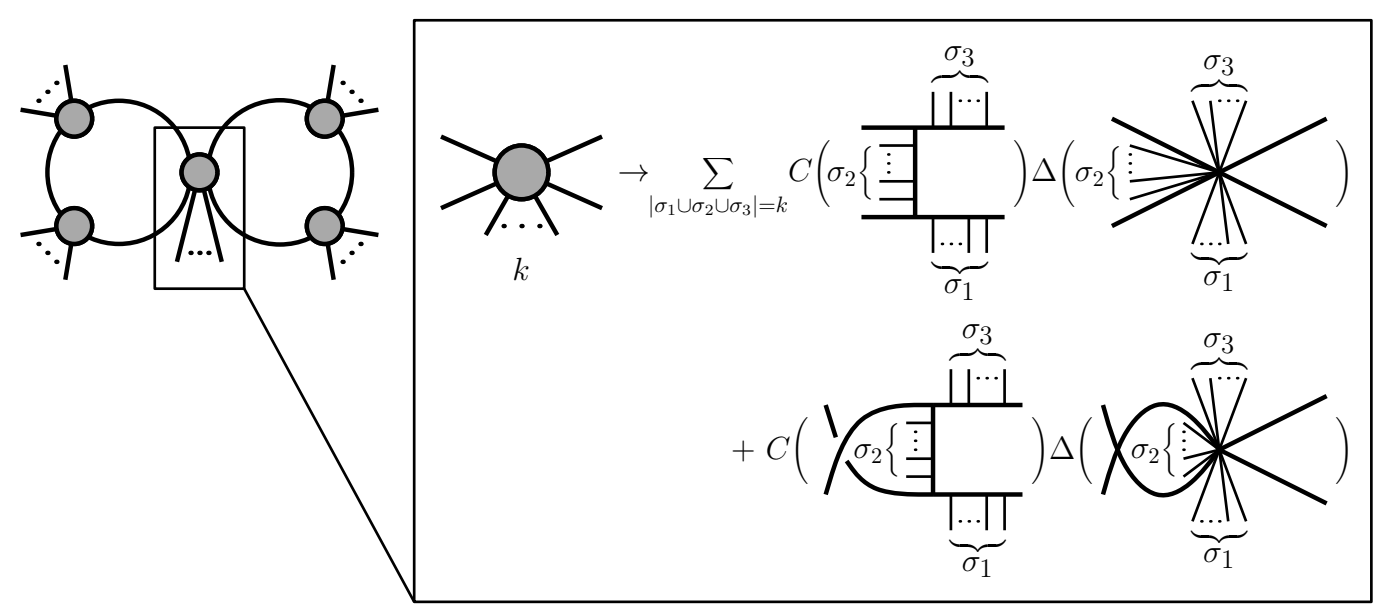

Figure 7. Inserting the DDM tree basis into a coloured cut of the butterfly topology. There are four loop edges, and the inset shows the result of inserting the DDM tree decomposition fixing the right ones. The sums run over the permutations of the external legs in the tree-level amplitude.

where we see the colour-ordered numerator $\Delta\left(\Psi^{-}\right)$. Its hierarchy subtraction naturally involves the numerator $\Delta(\square \nabla)$, which is excluded by the usual procedure in figure 6a in favour of its planar-looking orderings, with the underlying KK relation being

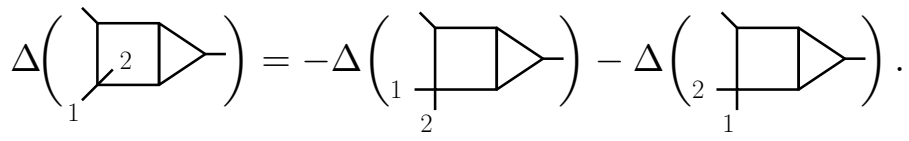

Therefore, one can perform the hierarchy subtraction for $\Delta\left(\Psi_{-}\right)$plugging in eq. (3.11) whenever $\Delta(\underline{L}-)$ is needed. To sum up, one must make sure to always KK-reduce the ancestor expressions to the basis of previously computed numerators $\Delta_{i}$. 


\subsubsection{Two-loop symmetry factors}

For coloured cuts with non-unity symmetry factors, one can often choose a KK-independent basis of colour-ordered cuts which is redundant under loop-momentum relabelling. Therefore, when this colour decomposition is projected onto irreducible numerators, which are momentum-routing-invariant under the integral sign, the basis is further reduced. We shall now demonstrate at two loops that in all cases where this is possible, the degree of the redundancy is equivalent to the symmetry factor, and many symmetry factors can be cancelled in this approach. However, the canonical choices in figures 6 and 7 do not always achieve this, in contrast to the one-loop case, where the usual stretch choice (shown in figure 5) is enough to find a simple momentum-routing redundant basis.

Consider the most symmetric case of the sunrise diagram $\Delta\left(\bigodot_{)}\right.$, which has a 3 ! permutation symmetry (or $(L+1)$ ! in the $L$-loop case of $\Delta\left(\bigodot_{)}\right)$). Evidently, the canonical DDM stretching procedure would single out two loop legs, so the full propagatorpermutation symmetry would not be accounted for. This is why in this case it is more useful to stretch across the two external legs on both trees. This results in $3 ! \cdot 3 !=36$ different terms, all of them being different permutations of the loop lines. However, there are only 6 distinct topologies, each appearing 6 times due to 3 ! different labellings of the propagators. As $\Delta_{i}$ are label-invariant, these terms can be collected, leading to an overall factor of 6 , cancelling the symmetry factor. Therefore, this procedure gives

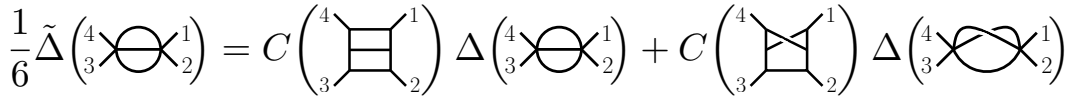

$$
\begin{aligned}
& +C\left({ }_{3}^{4} \mathcal{X}_{2}^{1}\right) \Delta\left({ }_{3}^{4} \bigotimes_{2}^{1}\right)+\{3 \leftrightarrow 4\} .
\end{aligned}
$$

This argument can be repeated for more than two external legs on the sides of the sunrisetype diagrams, in which case the permutation sum will still cancel the symmetry factor but contain ordered configurations of the extra external legs pointing inside the loops.

At two loops, there are six more graphs with non-unity symmetry factors listed in figure 8. All other such graphs should be topologically similar to these. For the planar and non-planar double triangle topologies, the factor due to the overall reflection symmetry of the diagrams, is cancelled by the same argument as for one-loop bubbles and tadpoles, through the canonical loop-leg stretching. In the case of the butterfly topologies, the canonical procedure in figures 6 and 7 results in a permutation sum over the internal loop edges, involving terms identical up to internal-edge relabelling. Once again this cancels the symmetry factors, leaving only two nonequivalent external leg configurations with doublebox colour factors in both cases.

To cancel the symmetry factor of the triangle-bubble, one may canonically DDMstretch the four-point vertices holding the legs of the bubble fixed,

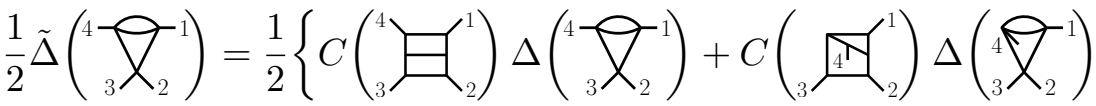

$$
\begin{aligned}
& \left.+C({ }_{3} \overbrace{41}) \Delta\left({ }_{3} \boldsymbol{X}_{2}\right)+C\left({ }_{3}^{4} \vec{r}_{2}\right) \Delta\left({ }_{3}^{4} \boldsymbol{X}_{2}\right)+\{2 \leftrightarrow 3\}\right\},
\end{aligned}
$$




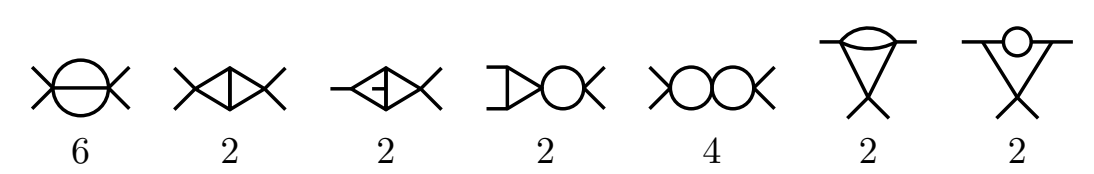

Figure 8. Example 4-point coloured diagrams and their associated symmetry factors. In all but one case these factors can be made to cancel in the colour decomposition.

with the vertex with legs 2 and 3 dealt with in the usual fashion. Now the symmetry factor can be removed because the graphs in the second line are equivalent to those shown in the first line due to the reflection symmetry of the vertices.

Finally, we should note that there is a special case where the symmetry factor cannot be cancelled - the diagram with the internal bubble associated with propagator renormalisation. As the symmetric part of the diagram is built out of three-point tree amplitudes, there is only one term arising from the colour decomposition, and so the symmetry factor remains. This is consistent with the fact that in this particular type of diagram the DDM-based colour factor respects the symmetry giving rise to that factor.

\subsubsection{Five-point two-loop amplitude}

Let us consider an explicit two-loop example in order to further elucidate the technique. Here we show the decomposition of the first two levels of the five-point pure Yang-Mills amplitude for arbitrary helicity:

$$
\begin{aligned}
& \mathcal{A}^{(2)}(1,2,3,4,5)=i g^{7} \sum_{\sigma \in S_{5}} \sigma \\
& \circ I\left[C ( { } _ { 4 } ^ { 5 } \square _ { 3 } ^ { 2 } ) \left\{\frac{1}{2} \Delta\left({ }_{4}^{5} \square_{3}^{2}\right)+\Delta\left({ }_{4}^{5} \square_{3}^{2} \square_{3}^{1}+\frac{1}{2} \Delta\left({ }_{4}^{5} \square_{3} \square_{2}^{1}\right)+\Delta\left({ }_{4}-\Sigma_{3}^{2}\right)\right.\right.\right.
\end{aligned}
$$

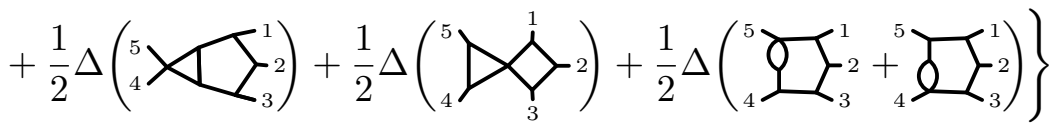

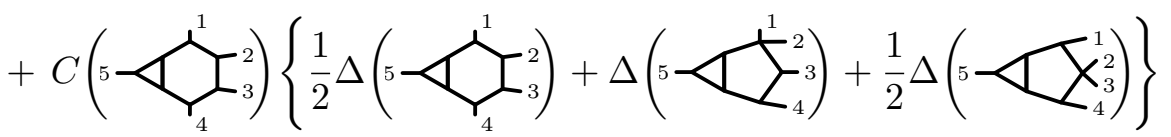

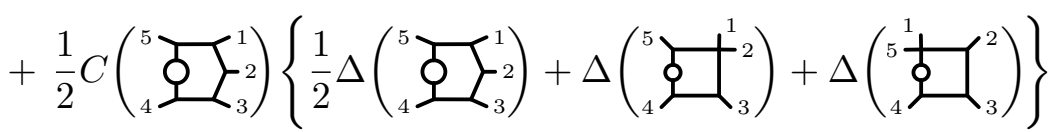

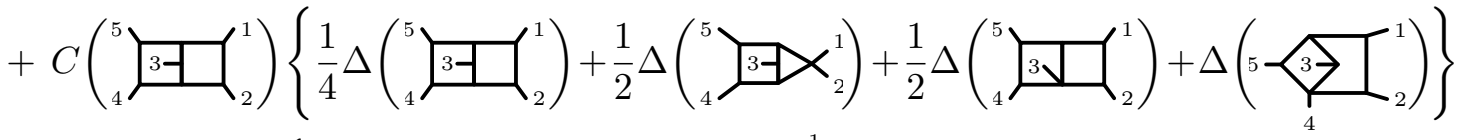

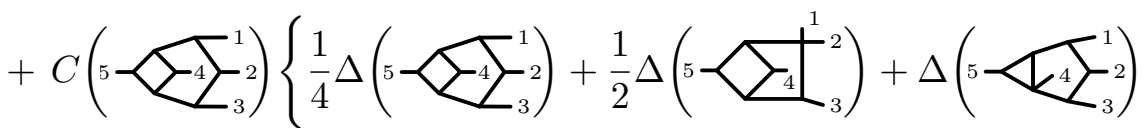

$$
\begin{aligned}
& \left.\left.+\frac{1}{2} \Delta\left({ }_{4} \boldsymbol{L}_{5}^{2}+{ }_{4} \delta^{5} \tau_{3}^{2}\right)\right\}+\ldots\right] \text {. }
\end{aligned}
$$

For the special case of all positive helicities, the corresponding irreducible numerators can be found in ref. [53], where the colour decomposition goes one level lower, but omits some 


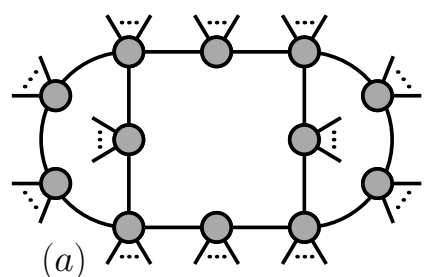

(a)

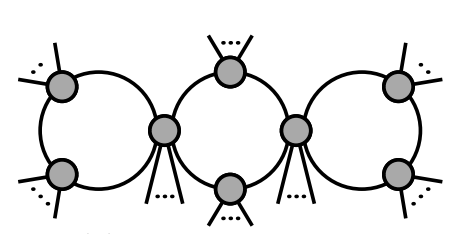

(e)

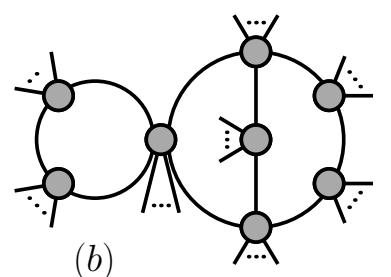

(b)
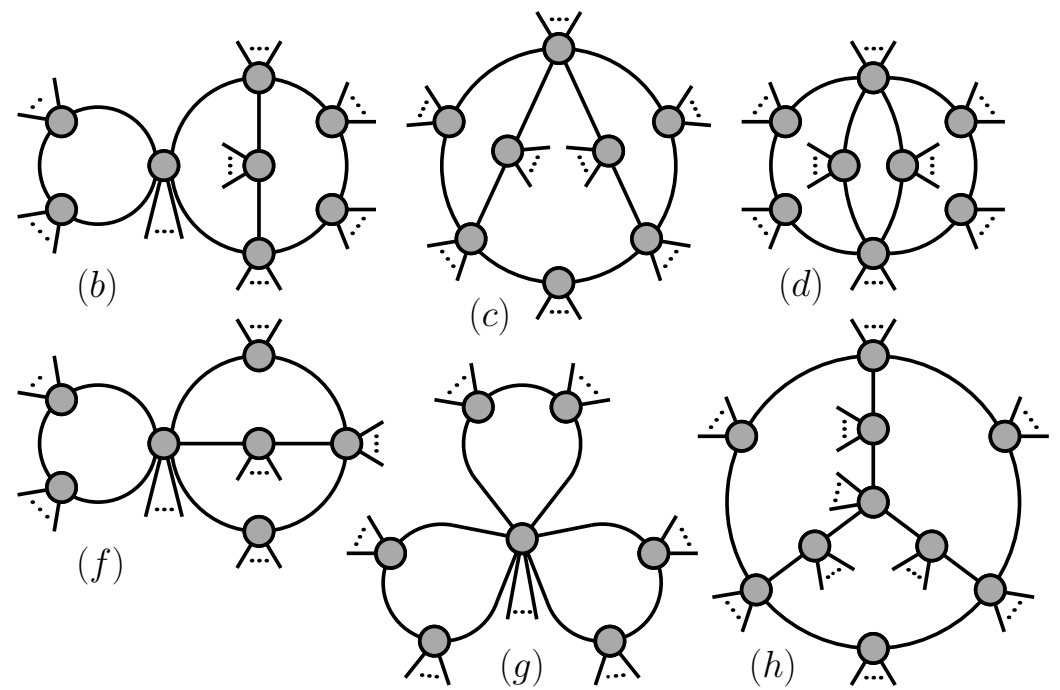

Figure 9. Basic topologies of three-loop coloured cuts.

topologies at the first two levels that are shown in eq. (3.14). Here we include all the topologies whose numerators do not have to vanish for arbitrary helicity. Going to the next level in full generality is straightforward but requires much more space than in the all-plus case [53].

Note that in eq. (3.14) the rational prefactors of the $\Delta \mathrm{s}$ are not symmetry factors, but factors that remove the overcounting by summing over the elements of $S_{5}$. This is true even for the diagrams that naively have symmetry factors (such as those with internal bubbles) because these can be cancelled in the decomposition, as explained in section 3.3.1. The only genuine symmetry factor due to bubble insertion is shown in front of the related colour factor.

A subtlety in the two-loop procedure comes from terms like $\Delta(\underline{I+}+\bar{l})$ and $\Delta(\Phi+$ E) - the cuts associated to these topologies do not exist. We have obtained their contribution to the decomposition (3.14) by taking the factorisation limit of the colour decomposition of the lower-level topology $\Delta(\bar{Y})$ which reveals the respective propagator, see appendix A for more details.

\subsection{Three-loop case}

At three loops, there are eight basic topologies of coloured cuts, they are shown in figure 9 . These involve vertices with up to six loop edges. In all cases the colour factors are generated by choosing two loop edges to fix and DDM-decompose, which gives colour-ordered orderings of the irreducible numerator with permutations of the external legs and the unfixed loop edges. In this way, the case of two loop edges has no ambiguity, as at one and two loops. Starting from the vertices with three loop legs, different choices of the two fixed loop edges produce different colour orderings and colour factors. For example, the central vertex of topology $(h)$ can be treated in three different ways, all of which correspond to some rotation of figure 10 . 


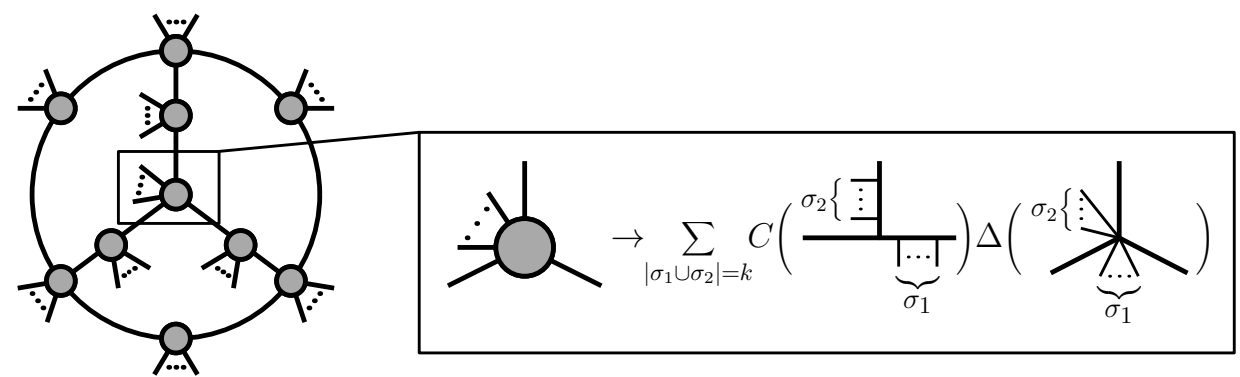

Figure 10. Inserting the DDM tree basis into the three-loop coloured cut of topology $(h)$. The inset treats the case of three loop-dependent legs in an analogous way to the inset (b) of figure 6 .

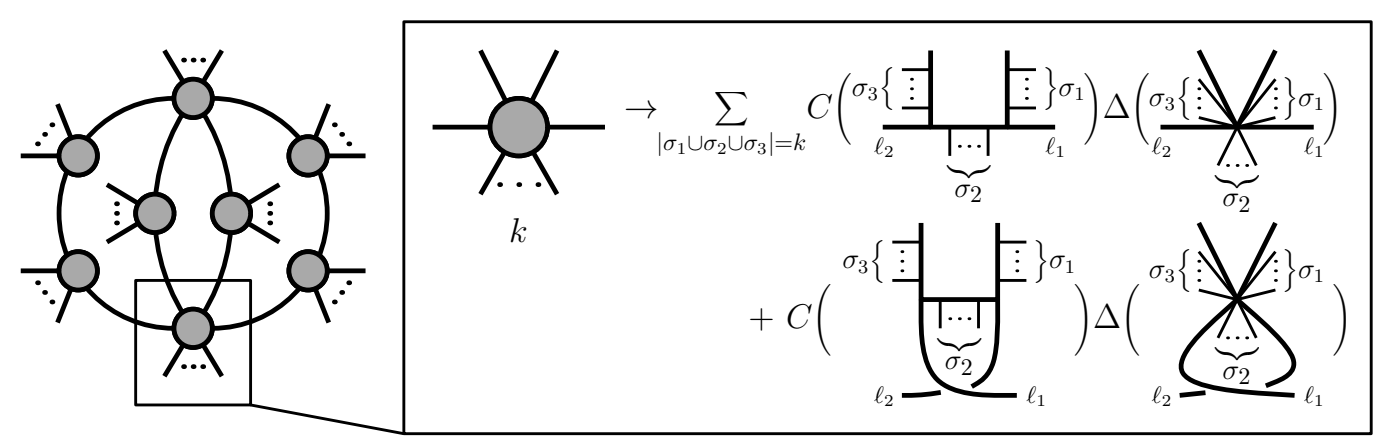

Figure 11. Inserting the DDM tree basis into the three-loop coloured cut of topology $(d)$. The inset treats the case of four loop-dependent legs and shows the two permutations of the loop legs labelled $\ell_{1}$ and $\ell_{2}$, which is analogous to figure 7 .

It is preferable to pick the fixed loop legs with regard to the symmetry of the topology. For instance, topology $(d)$ contains two vertices with four loop-dependent edges, and the four branches can be considered equivalent for the unordered graph. However, the colour decomposition makes better use of the specifics of that topology if the upper vertex is treated by fixing the same two loop branches that are fixed in the treatment of the lower vertex shown in figure 11.

The vertices with five and six loop-dependent legs occur only in topologies $(f)$ and $(g)$ and are treated in figures 12 and 13. The corresponding permutations involve three and four unfixed loop legs, respectively, and thus generate 6 and 24 ordered topologies. For simplicity, only the first couple of them are depicted.

\subsubsection{Four-point three-loop example}

As a simple example of the three-loop colour decomposition, we consider the four-gluon amplitude in $\mathcal{N}=4$ supersymmetric Yang-Mills theory, extensively studied in the literature at the integrand level [79-85], as well as recently integrated in ref. [86].

For convenience, it is commonplace to encode all the helicity configurations of the external states inside the overall kinematic prefactor

$$
\mathcal{K}=i s_{12} s_{14} A_{4}^{(0)}(1,2,3,4)
$$




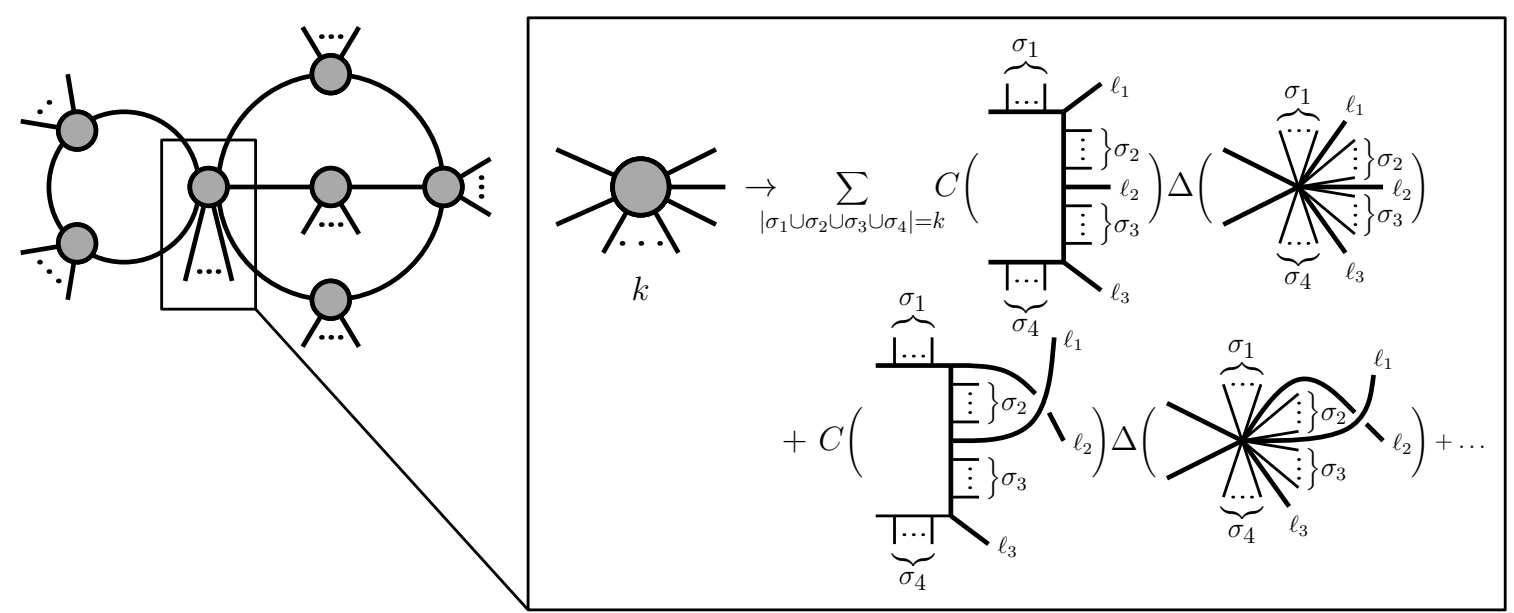

Figure 12. Inserting the DDM tree basis into the three-loop coloured cut of topology $(f)$. The inset treats the case of five loop-dependent legs and shows explicitly two out of 6 permutations of the loop legs labelled $\ell_{1}, \ell_{2}$ and $\ell_{3}$.

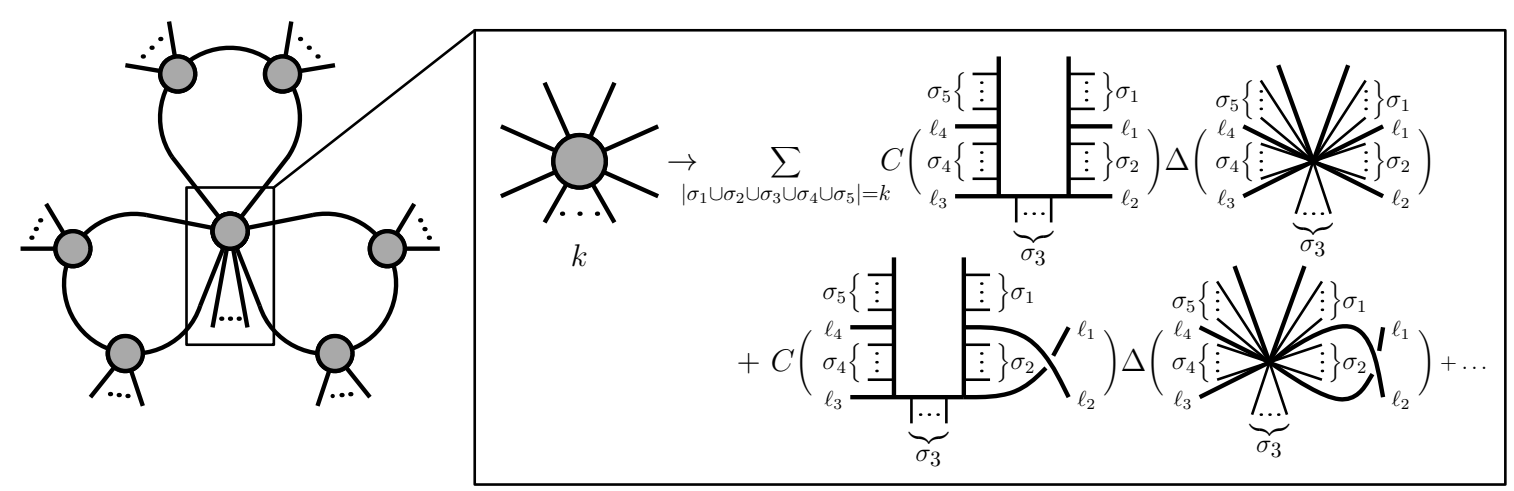

Figure 13. Inserting the DDM tree basis into the three-loop coloured cut of topology $(g)$. The inset treats the case of six loop-dependent legs and shows explicitly two out of 24 permutations of the loop legs labelled $\ell_{1}, \ell_{2}, \ell_{3}$ and $\ell_{4}$.

The tree-level colour-ordered amplitude to which it is proportional can be understood either as a super-amplitude or any one of its nonzero MHV components.

The internal-momentum parametrisation of the irreducible numerators is chosen restricting to irreducible scalar products (ISPs) purely linear in loop momenta. ${ }^{6}$ Moreover, we choose the basis elements in such a way that the resulting numerators respect the symmetries of their topologies, which often [54] makes their treatment easier. We find

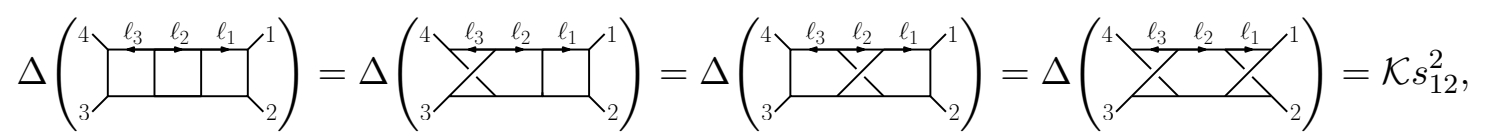

\footnotetext{
${ }^{6}$ Here and below, we used the automated Mathematica package BasisDet [48] to verify irreducibility.
} 


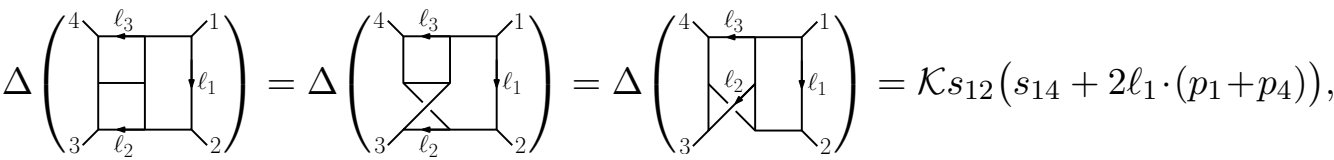

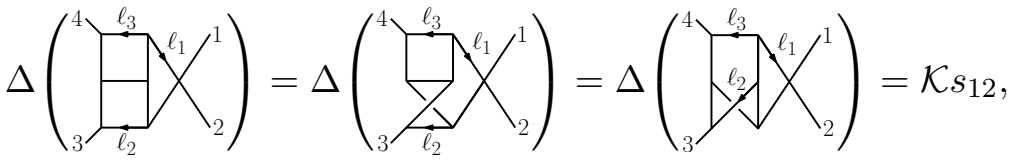

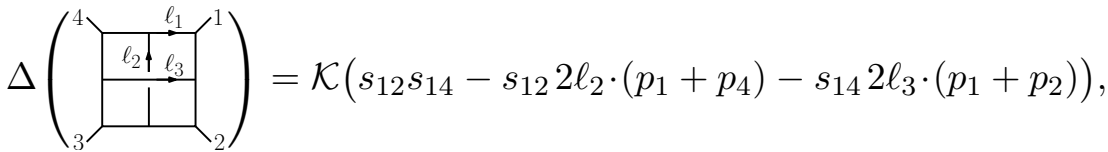

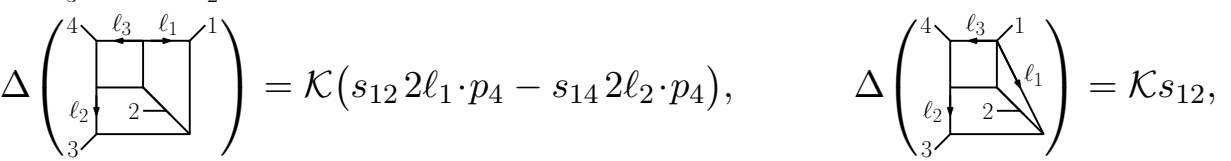

$$
\begin{aligned}
& \Delta\left(\sum_{\ell_{2}}^{{ }_{2}} Y_{3}^{\ell_{3}} T^{\ell_{1}}{ }^{1}\right)=\mathcal{K}\left(2 s_{14}-s_{12}-s_{13}\right) / 3
\end{aligned}
$$

where we have shown only non-vanishing topologies. Note that the numerators $(3.16 \mathrm{~b})$ contain a reducible scalar product $\left(\ell_{1} \cdot p_{1}\right)=\ell_{1}^{2}-\left(\ell_{1}-p_{1}\right)^{2}$, but it is combined together with the irreducible $\left(\ell_{1} \cdot p_{4}\right)$ into an ISP that respects the flip symmetry $\left(\ell_{1} \cdot\left(p_{1}+p_{4}\right)\right)=$ $\left(-\ell_{1} \cdot\left(p_{2}+p_{3}\right)\right)$ of the first and the second topologies in eq. (3.16b).

The full amplitude for arbitrary helicity states can now be constructed from the irreducible numerators (3.16),

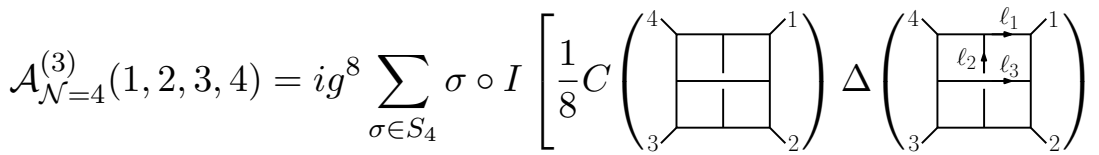

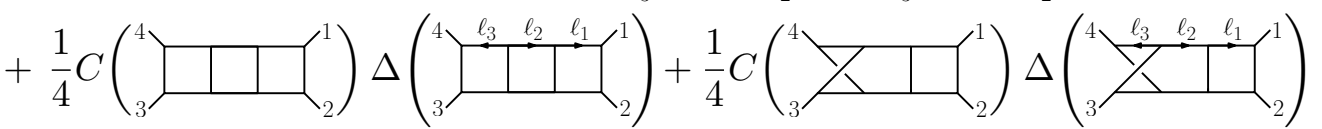

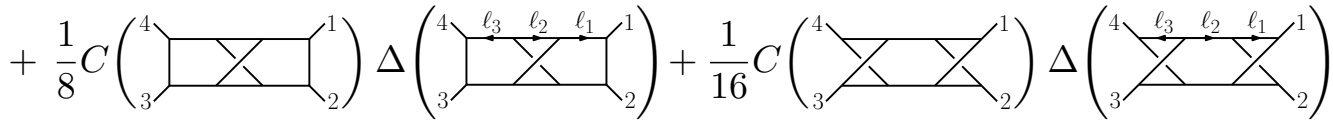

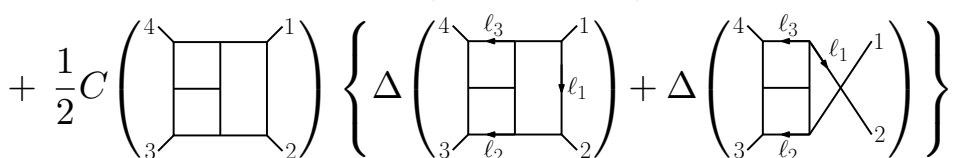

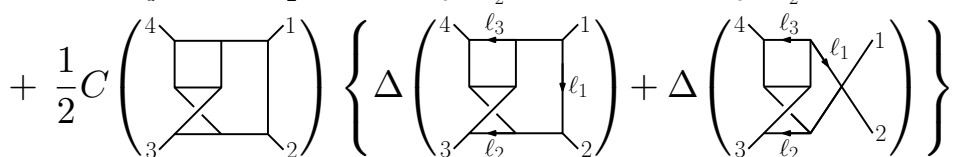

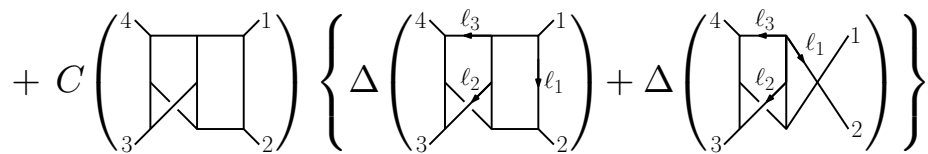

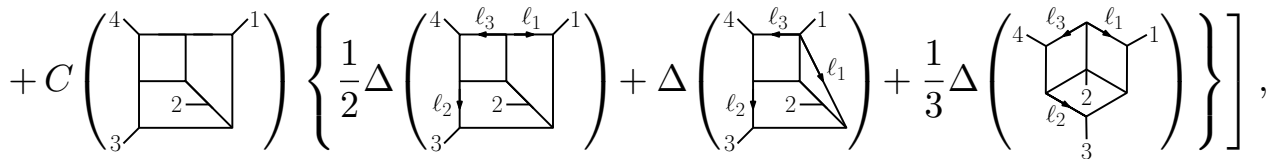


where the numerical prefactors are there just to remove overcounting caused by the full overall permutation sum, except for the fifth diagram which has a genuine symmetry factor 2. All colour factors are cubic, both for the top-level and lower-level numerators. The most subtle colour decomposition here occurs in the last line. The DDM-based decomposition for the colour-dressed version of the topology (3.16f) is

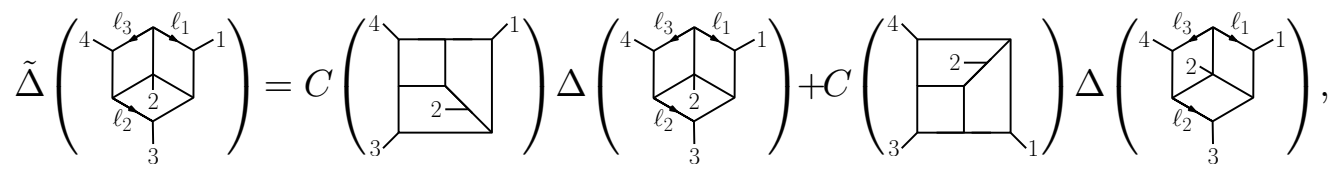

where we "stretched" the numerator diagram by its top and right internal "spokes" analogously to the inset (b) of figure 6. We could have just as well picked two other pairs of internal propagators. Regardless, the minimal sum should include eq. (3.18) and its three other non-equivalent permutations, corresponding to legs 2, 1, 3 and 4 chosen in the middle of the diagram. The overall permutation sum in eq. (3.17) is therefore overcomplete and effectively symmetrises over the three choices of internal propagators to "stretch" by in order to decompose the colour-dressed numerator (3.18).

In appendix B we explicitly map our colour decomposition (3.17) to the cubic-graph decomposition found in refs. [79, 80, 82].

\section{Summary and outlook}

We have discussed a method to promote tree-level colour decompositions to loop level in the framework of integrand reduction via unitarity cuts. Effectively, one takes a colour decomposition of a coloured cut and promotes it to a colour decomposition of the associated irreducible numerators. This can be achieved with any given colour decomposition, but is most powerful when applied to one which is KK-independent. We have shown that colourordered irreducible numerators can be chosen to inherit KK relations of their associated cuts and demonstrated how this can be used to simplify amplitude calculations in gauge theories with various degrees of supersymmetry. As the irreducible numerators live under an integral sign, different momentum reroutings of the same topology are equivalent, and this can used to cancel the symmetry factors, at least at one and two loops. At one loop, this allows the method to recover the DDM one-loop decomposition [66]. At two loops, we have supplemented the five-point colour decomposition of ref. [54] by the maximal and next-to-maximal topologies that correspond to vanishing cuts for the all positive external helicities but should be included in other helicity configurations. As a three-loop example, we have also considered the four-point amplitude in maximally supersymmetry Yang-Mills theory [79]. More generally, we have shown how the DDM-based colour decomposition applies to any type of three-loop cut topology.

In this note, we considered the multi-loop integrand construction using irreducible numerators. We have assumed that the numerator KK relations hold at the integrand level, which can be helped by a loop-momentum parametrisation of a numerator that is tailored to its symmetries. However, this assumption can be removed provided that the integrated amplitude stays the same or, equivalently, it satisfies all the unitarity cuts. 
Hence there should be a way to use the presented colour decomposition in absence of off-shell KK relations, though self-consistency of the hierarchy subtraction may be more difficult to achieve in this case. For instance, this may be relevant in the framework of non-planar on-shell diagrams [85, 87-92], where the requirement of the irreducibility is replaced by a connection to the amplituhedron structure $[93,94]$. It would be interesting to use the presented colour decomposition in this setting.

Moreover, in future work we hope to further explore this technique in the context of QCD amplitudes with quarks [95-98]. Indeed, the recent studies of the tree-level KK relations for multi-quark amplitudes [99-103], have led to a proper colour decomposition [104, 105] of a quark-gluon amplitude into KK-independent basis of partial amplitudes [101, 102], analogous in this respect to the DDM formula [66]. In this way, the presented method will be applicable to a greater variety of phenomenologically pertinent amplitude calculations.

\section{Acknowledgments}

We are very grateful to Simon Badger, Fernando Febres-Cordero, Harald Ita, Gustav Mogull and Donal O'Connell for enlightening discussions and for collaboration on related topics. We thank Harald Ita and Donal O'Connell for useful comments on the draft, as well as Samuel Abreu, Matthieu Jaquier, Tiziano Peraro and Christopher Schwan for helpful discussions. AO is supported in part by the Marie Curie FP7 grant 631370. BP is supported by the Alexander von Humboldt Foundation, in the framework of the Sofja Kovalevskaja Award 2014, endowed by the German Federal Ministry of Education and Research. AO thanks Nordita for hospitality during the programme "Aspects of Amplitudes."

\section{A Bubble-insertion subtlety}

In eq. (3.14) we used the factorisation limit of the following relation:

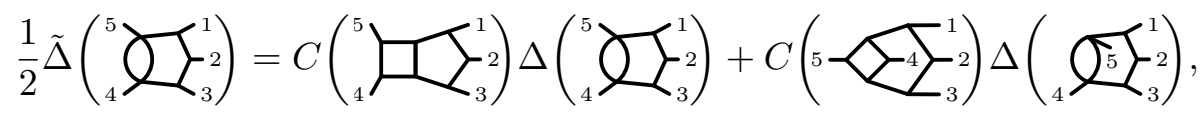

which is assumed to hold under the integration sign similarly to the result of eq. (3.13). This implicitly relies on the KK relations for the colour-ordered numerators and corresponding cuts, such as

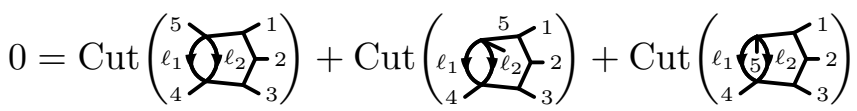

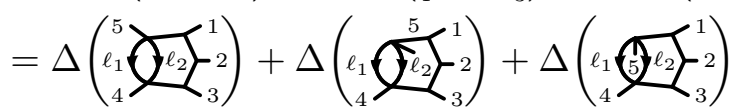

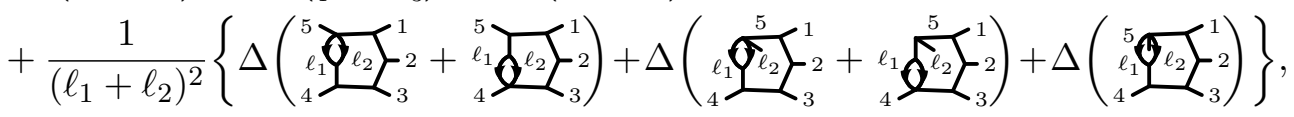

where all the other higher-level topologies cancel in the usual way. The subtlety here is that the topologies like do not correspond to well-defined cuts. Instead, we understand 
the numerator $\Delta(\underline{Y}+\bar{\zeta})$ as a single coefficient function of the $1 /\left(\ell_{1}+\ell_{2}\right)^{2}$ pole inside the finite $\mathrm{Cut}\left(\mathcal{Y}_{)}\right)$. This prevents us from separating and cancelling the graphs in the last line of eq. (A.2), as it would normally happen. Therefore, eq. (A.2) implies not only the KK relation between $\Delta(\mathcal{K}), \Delta(\sigma)$ and $\Delta(\sigma)$ but also an accompanying KK relation of less familiar form:

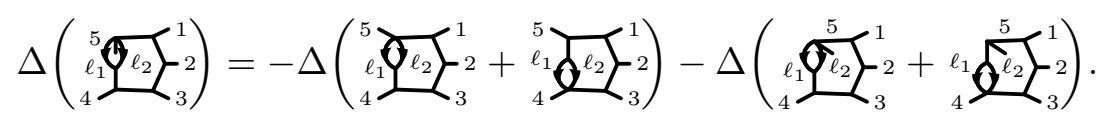

\section{B Check of four-point three-loop example}

Here let us show how our amplitude decomposition (3.17) relates to the cubic-graph decomposition found in refs. [79, 80, 82]:

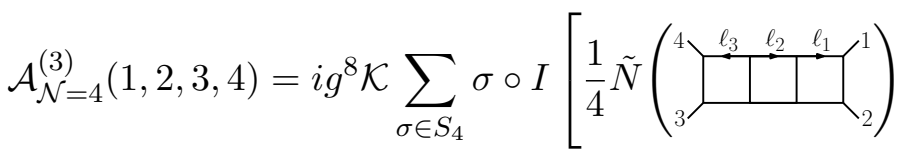

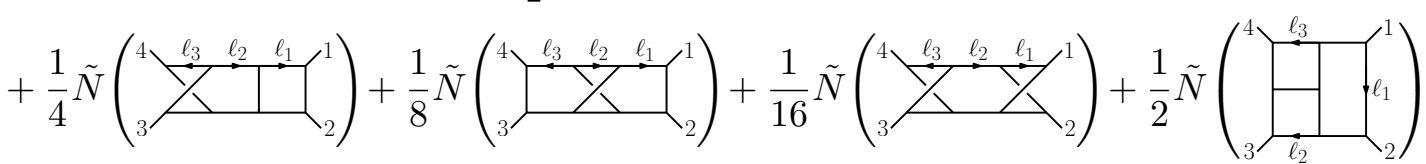

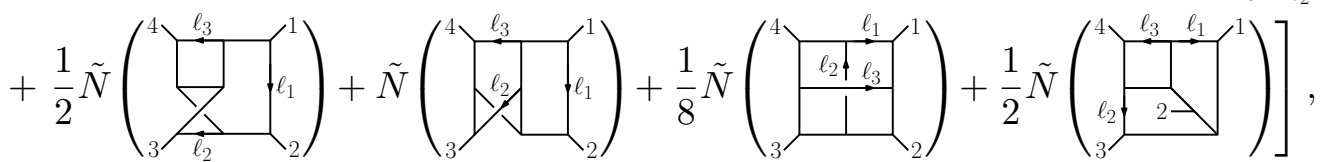

where the purely trivalent colour factors are implicit, and the kinematic parts of the numerators are given by

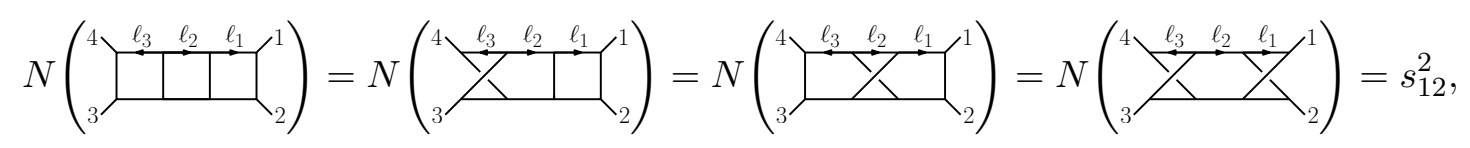

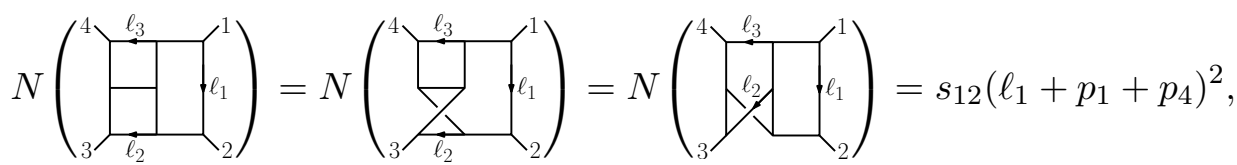

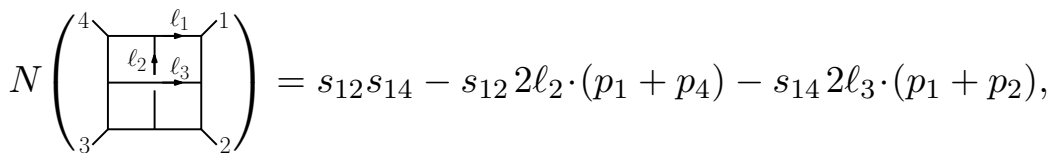

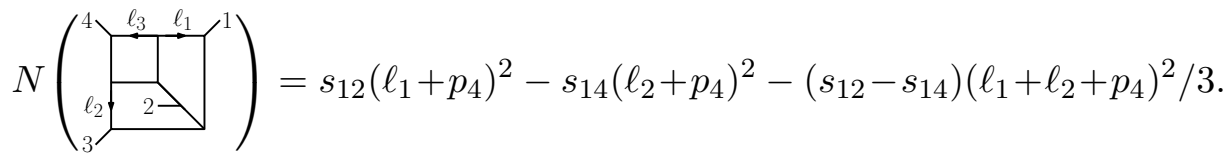

Here the numerators (B.2a) and (B.2c) equal already their irreducible counterparts $(3.16 \mathrm{a})$ and $(3.16 \mathrm{~d})$ (up to the prefactor $\mathcal{K}$ ). The numerators (B.2b) are naturally combined from their irreducible counterparts (3.16a) and related lower-level 
topologies (3.16c), i.e.

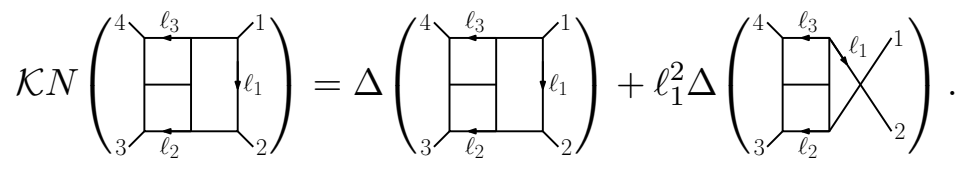

Finally, to obtain the numerator (B.2d), we can use the diagonal-flip antisymmetry of the colour factor in the last line of eq. (3.17),

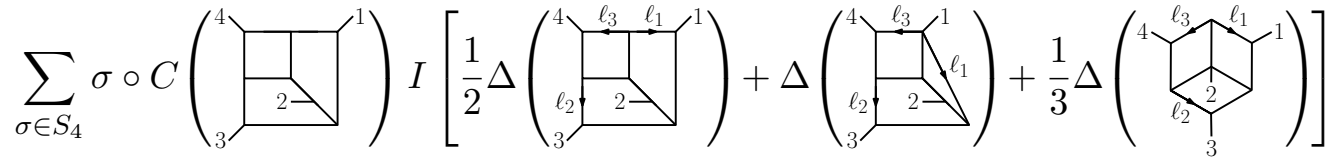

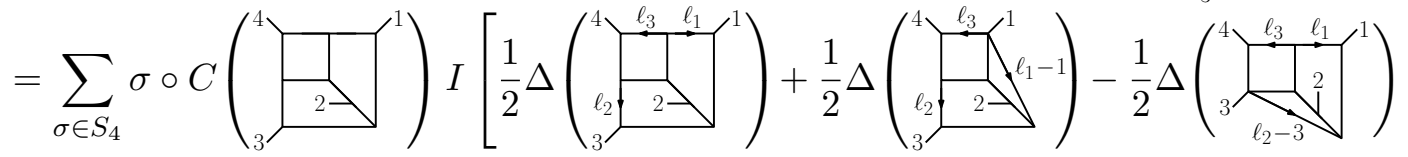

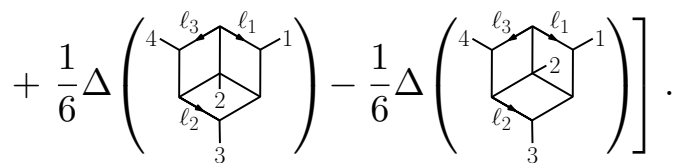

Taking into account that our integration measure (2.22) includes topology-specific propagators, we retrieve precisely

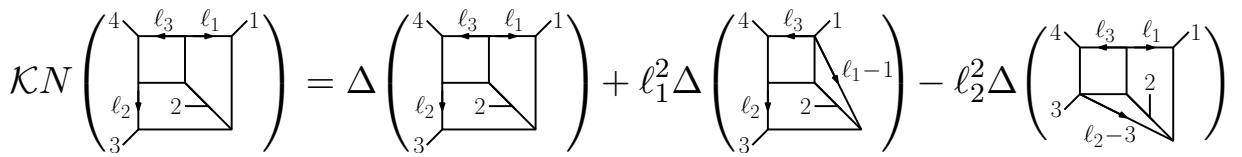

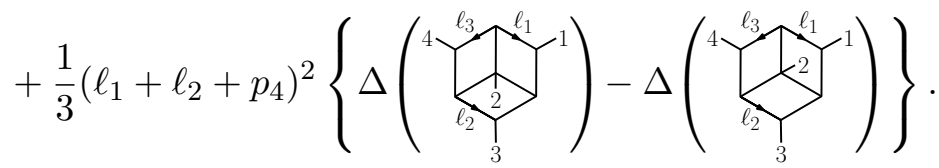

In this way, we map to our irreducible-numerator decomposition (3.17) to the cubic-graph decomposition (B.1). It should be noted that one can in principle choose irreducible monomials in such a way that only the top-level numerators (B.2) are left non-vanishing. In section 3.4.1 we intentionally restricted ourselves to the parametrisation at most linear in loop momenta in order to demonstrate nontrivial elements of the DDM-based colour decomposition.

Open Access. This article is distributed under the terms of the Creative Commons Attribution License (CC-BY 4.0), which permits any use, distribution and reproduction in any medium, provided the original author(s) and source are credited.

\section{References}

[1] C. Anastasiou, E.W.N. Glover, C. Oleari and M.E. Tejeda-Yeomans, Two-loop QCD corrections to the scattering of massless distinct quarks, Nucl. Phys. B 601 (2001) 318 [hep-ph/0010212] [INSPIRE].

[2] C. Anastasiou, E.W.N. Glover, C. Oleari and M.E. Tejeda-Yeomans, Two loop QCD corrections to massless identical quark scattering, Nucl. Phys. B 601 (2001) 341 [hep-ph/0011094] [INSPIRE]. 
[3] C. Anastasiou, E.W.N. Glover, C. Oleari and M.E. Tejeda-Yeomans, Two loop QCD corrections to massless quark gluon scattering, Nucl. Phys. B 605 (2001) 486 [hep-ph/0101304] [INSPIRE].

[4] E.W.N. Glover, C. Oleari and M.E. Tejeda-Yeomans, Two loop QCD corrections to gluon-gluon scattering, Nucl. Phys. B 605 (2001) 467 [hep-ph/0102201] [INSPIRE].

[5] L.W. Garland, T. Gehrmann, E.W.N. Glover, A. Koukoutsakis and E. Remiddi, The Two loop QCD matrix element for $e^{+} e^{-} \rightarrow 3$ jets, Nucl. Phys. B 627 (2002) 107 [hep-ph/0112081] [INSPIRE].

[6] L.W. Garland, T. Gehrmann, E.W.N. Glover, A. Koukoutsakis and E. Remiddi, Two loop QCD helicity amplitudes for $e^{+} e^{-} \rightarrow 3$ jets, Nucl. Phys. B 642 (2002) 227 [hep-ph/0206067] [INSPIRE].

[7] S. Catani, L. Cieri, D. de Florian, G. Ferrera and M. Grazzini, Diphoton production at hadron colliders: a fully-differential QCD calculation at NNLO, Phys. Rev. Lett. 108 (2012) 072001 [arXiv: 1110.2375] [INSPIRE].

[8] T. Gehrmann, M. Jaquier, E.W.N. Glover and A. Koukoutsakis, Two-Loop QCD Corrections to the Helicity Amplitudes for $H \rightarrow 3$ partons, JHEP 02 (2012) 056 [arXiv:1112.3554] [INSPIRE].

[9] M. Czakon, P. Fiedler and A. Mitov, Total Top-Quark Pair-Production Cross section at Hadron Colliders Through $O\left(\alpha_{S}^{4}\right)$, Phys. Rev. Lett. 110 (2013) 252004 [arXiv:1303.6254] [INSPIRE].

[10] M. Grazzini, S. Kallweit, D. Rathlev and A. Torre, $Z \gamma$ production at hadron colliders in NNLO QCD, Phys. Lett. B 731 (2014) 204 [arXiv:1309.7000] [INSPIRE].

[11] F. Cascioli et al., ZZ production at hadron colliders in NNLO QCD, Phys. Lett. B 735 (2014) 311 [arXiv: 1405.2219] [INSPIRE].

[12] T. Gehrmann et al., $W^{+} W^{-}$Production at Hadron Colliders in Next to Next to Leading Order QCD, Phys. Rev. Lett. 113 (2014) 212001 [arXiv: 1408.5243] [INSPIRE].

[13] X. Chen, T. Gehrmann, E.W.N. Glover and M. Jaquier, Precise QCD predictions for the production of Higgs + jet final states, Phys. Lett. B 740 (2015) 147 [arXiv:1408.5325] [INSPIRE].

[14] F. Caola, J.M. Henn, K. Melnikov, A.V. Smirnov and V.A. Smirnov, Two-loop helicity amplitudes for the production of two off-shell electroweak bosons in quark-antiquark collisions, JHEP 11 (2014) 041 [arXiv:1408.6409] [INSPIRE].

[15] M. Czakon, P. Fiedler and A. Mitov, Resolving the Tevatron Top Quark Forward-Backward Asymmetry Puzzle: Fully Differential Next-to-Next-to-Leading-Order Calculation, Phys. Rev. Lett. 115 (2015) 052001 [arXiv: 1411. 3007] [INSPIRE].

[16] T. Gehrmann, A. von Manteuffel and L. Tancredi, The two-loop helicity amplitudes for $q \bar{q}^{\prime} \rightarrow V_{1} V_{2} \rightarrow 4$ leptons, JHEP 09 (2015) 128 [arXiv: 1503.04812] [INSPIRE].

[17] F. Caola, J.M. Henn, K. Melnikov, A.V. Smirnov and V.A. Smirnov, Two-loop helicity amplitudes for the production of two off-shell electroweak bosons in gluon fusion, JHEP 06 (2015) 129 [arXiv: 1503.08759] [INSPIRE].

[18] A. von Manteuffel and L. Tancredi, The two-loop helicity amplitudes for $g g \rightarrow V_{1} V_{2} \rightarrow 4$ leptons, JHEP 06 (2015) 197 [arXiv:1503.08835] [INSPIRE]. 
[19] M. Grazzini, S. Kallweit and D. Rathlev, $W \gamma$ and $Z \gamma$ production at the LHC in NNLO QCD, JHEP 07 (2015) 085 [arXiv: 1504.01330] [INSPIRE].

[20] R. Boughezal, C. Focke, X. Liu and F. Petriello, $W$-boson production in association with a jet at next-to-next-to-leading order in perturbative QCD, Phys. Rev. Lett. 115 (2015) 062002 [arXiv: 1504.02131] [INSPIRE].

[21] R. Boughezal, F. Caola, K. Melnikov, F. Petriello and M. Schulze, Higgs boson production in association with a jet at next-to-next-to-leading order, Phys. Rev. Lett. 115 (2015) 082003 [arXiv: 1504.07922] [inSPIRE].

[22] R. Boughezal, C. Focke, W. Giele, X. Liu and F. Petriello, Higgs boson production in association with a jet at NNLO using jettiness subtraction, Phys. Lett. B 748 (2015) 5 [arXiv: 1505.03893] [INSPIRE].

[23] A. Gehrmann-De Ridder, T. Gehrmann, E.W.N. Glover, A. Huss and T.A. Morgan, Precise $Q C D$ predictions for the production of a $Z$ boson in association with a hadronic jet, Phys. Rev. Lett. 117 (2016) 022001 [arXiv:1507.02850] [INSPIRE].

[24] C. Anastasiou, C. Duhr, F. Dulat, F. Herzog and B. Mistlberger, Higgs Boson Gluon-Fusion Production in QCD at Three Loops, Phys. Rev. Lett. 114 (2015) 212001 [arXiv: 1503.06056] [INSPIRE].

[25] Z. Bern, L.J. Dixon, D.C. Dunbar and D.A. Kosower, One loop $n$ point gauge theory amplitudes, unitarity and collinear limits, Nucl. Phys. B 425 (1994) 217 [hep-ph/9403226] [INSPIRE].

[26] Z. Bern, L.J. Dixon, D.C. Dunbar and D.A. Kosower, Fusing gauge theory tree amplitudes into loop amplitudes, Nucl. Phys. B 435 (1995) 59 [hep-ph/9409265] [INSPIRE].

[27] R. Britto, F. Cachazo and B. Feng, Generalized unitarity and one-loop amplitudes in $N=4$ super-Yang-Mills, Nucl. Phys. B 725 (2005) 275 [hep-th/0412103] [InSPIRE].

[28] D. Forde, Direct extraction of one-loop integral coefficients, Phys. Rev. D 75 (2007) 125019 [arXiv:0704.1835] [INSPIRE].

[29] R. Britto, E. Buchbinder, F. Cachazo and B. Feng, One-loop amplitudes of gluons in SQCD, Phys. Rev. D 72 (2005) 065012 [hep-ph/0503132] [INSPIRE].

[30] C. Anastasiou, R. Britto, B. Feng, Z. Kunszt and P. Mastrolia, D-dimensional unitarity cut method, Phys. Lett. B 645 (2007) 213 [hep-ph/0609191] [INSPIRE].

[31] W.T. Giele, Z. Kunszt and K. Melnikov, Full one-loop amplitudes from tree amplitudes, JHEP 04 (2008) 049 [arXiv:0801.2237] [INSPIRE].

[32] Z. Bern et al., Next-to-Leading Order W+5-Jet Production at the LHC, Phys. Rev. D 88 (2013) 014025 [arXiv: 1304.1253] [INSPIRE].

[33] S. Badger, B. Biedermann, P. Uwer and V. Yundin, Next-to-leading order QCD corrections to five jet production at the LHC, Phys. Rev. D 89 (2014) 034019 [arXiv:1309.6585] [INSPIRE].

[34] D.A. Kosower and K.J. Larsen, Maximal Unitarity at Two Loops, Phys. Rev. D 85 (2012) 045017 [arXiv: 1108.1180] [inSPIRE].

[35] S. Caron-Huot and K.J. Larsen, Uniqueness of two-loop master contours, JHEP 10 (2012) 026 [arXiv: 1205.0801] [INSPIRE]. 
[36] H. Johansson, D.A. Kosower and K.J. Larsen, Two-Loop Maximal Unitarity with External Masses, Phys. Rev. D 87 (2013) 025030 [arXiv:1208.1754] [InSPIRE].

[37] H. Johansson, D.A. Kosower and K.J. Larsen, Maximal Unitarity for the Four-Mass Double Box, Phys. Rev. D 89 (2014) 125010 [arXiv:1308.4632] [inSPIRE].

[38] M. Søgaard, Global Residues and Two-Loop Hepta-Cuts, JHEP 09 (2013) 116 [arXiv: 1306.1496] [INSPIRE].

[39] M. Søgaard and Y. Zhang, Multivariate Residues and Maximal Unitarity, JHEP 12 (2013) 008 [arXiv: 1310.6006] [INSPIRE].

[40] M. Søgaard and Y. Zhang, Unitarity Cuts of Integrals with Doubled Propagators, JHEP 07 (2014) 112 [arXiv: 1403.2463] [INSPIRE].

[41] M. Søgaard and Y. Zhang, Massive Nonplanar Two-Loop Maximal Unitarity, JHEP 12 (2014) 006 [arXiv: 1406.5044] [InSPIRE].

[42] M. Søgaard and Y. Zhang, Elliptic Functions and Maximal Unitarity, Phys. Rev. D 91 (2015) 081701 [arXiv: 1412.5577] [INSPIRE].

[43] H. Johansson, D.A. Kosower, K.J. Larsen and M. Søgaard, Cross-Order Integral Relations from Maximal Cuts, Phys. Rev. D 92 (2015) 025015 [arXiv: 1503.06711] [INSPIRE].

[44] P. Mastrolia and G. Ossola, On the Integrand-Reduction Method for Two-Loop Scattering Amplitudes, JHEP 11 (2011) 014 [arXiv:1107.6041] [INSPIRE].

[45] S. Badger, H. Frellesvig and Y. Zhang, Hepta-Cuts of Two-Loop Scattering Amplitudes, JHEP 04 (2012) 055 [arXiv: 1202.2019] [INSPIRE].

[46] H. Ita, Two-loop Integrand Decomposition into Master Integrals and Surface Terms, Phys. Rev. D 94 (2016) 116015 [arXiv: 1510.05626] [INSPIRE].

[47] G. Ossola, C.G. Papadopoulos and R. Pittau, Reducing full one-loop amplitudes to scalar integrals at the integrand level, Nucl. Phys. B 763 (2007) 147 [hep-ph/0609007] [INSPIRE].

[48] Y. Zhang, Integrand-Level Reduction of Loop Amplitudes by Computational Algebraic Geometry Methods, JHEP 09 (2012) 042 [arXiv:1205.5707] [INSPIRE].

[49] P. Mastrolia, E. Mirabella, G. Ossola and T. Peraro, Scattering Amplitudes from Multivariate Polynomial Division, Phys. Lett. B 718 (2012) 173 [arXiv:1205.7087] [INSPIRE].

[50] S. Badger, H. Frellesvig and Y. Zhang, An Integrand Reconstruction Method for Three-Loop Amplitudes, JHEP 08 (2012) 065 [arXiv:1207.2976] [INSPIRE].

[51] P. Mastrolia, E. Mirabella, G. Ossola and T. Peraro, Integrand-Reduction for Two-Loop Scattering Amplitudes through Multivariate Polynomial Division, Phys. Rev. D 87 (2013) 085026 [arXiv: 1209.4319] [INSPIRE].

[52] P. Mastrolia, E. Mirabella, G. Ossola and T. Peraro, Multiloop Integrand Reduction for Dimensionally Regulated Amplitudes, Phys. Lett. B 727 (2013) 532 [arXiv:1307.5832] [INSPIRE].

[53] S. Badger, H. Frellesvig and Y. Zhang, A Two-Loop Five-Gluon Helicity Amplitude in QCD, JHEP 12 (2013) 045 [arXiv: 1310.1051] [INSPIRE].

[54] S. Badger, G. Mogull, A. Ochirov and D. O'Connell, A Complete Two-Loop, Five-Gluon Helicity Amplitude in Yang-Mills Theory, JHEP 10 (2015) 064 [arXiv:1507.08797] [INSPIRE]. 
[55] S. Badger, G. Mogull and T. Peraro, Local integrands for two-loop all-plus Yang-Mills amplitudes, JHEP 08 (2016) 063 [arXiv: 1606.02244] [INSPIRE].

[56] T. Gehrmann, J.M. Henn and N.A. Lo Presti, Analytic form of the two-loop planar five-gluon all-plus-helicity amplitude in QCD, Phys. Rev. Lett. 116 (2016) 062001 [Erratum ibid. 116 (2016) 189903] [arXiv: 1511.05409] [INSPIRE].

[57] D.C. Dunbar and W.B. Perkins, Two-loop five-point all plus helicity Yang-Mills amplitude, Phys. Rev. D 93 (2016) 085029 [arXiv:1603.07514] [INSPIRE].

[58] D.C. Dunbar, G.R. Jehu and W.B. Perkins, The two-loop n-point all-plus helicity amplitude, Phys. Rev. D 93 (2016) 125006 [arXiv:1604.06631] [INSPIRE].

[59] D.C. Dunbar, G.R. Jehu and W.B. Perkins, Two-loop six gluon all plus helicity amplitude, Phys. Rev. Lett. 117 (2016) 061602 [arXiv: 1605.06351] [INSPIRE].

[60] Z. Bern, J.S. Rozowsky and B. Yan, Two loop four gluon amplitudes in $N=4$ super Yang-Mills, Phys. Lett. B 401 (1997) 273 [hep-ph/9702424] [INSPIRE].

[61] Z. Bern, A. De Freitas and L.J. Dixon, Two loop helicity amplitudes for gluon-gluon scattering in QCD and supersymmetric Yang-Mills theory, JHEP 03 (2002) 018 [hep-ph/0201161] [INSPIRE].

[62] S.G. Naculich, All-loop group-theory constraints for color-ordered SU(N) gauge-theory amplitudes, Phys. Lett. B 707 (2012) 191 [arXiv:1110.1859] [INSPIRE].

[63] A.C. Edison and S.G. Naculich, $\mathrm{SU}(N)$ group-theory constraints on color-ordered five-point amplitudes at all loop orders, Nucl. Phys. B 858 (2012) 488 [arXiv:1111.3821] [InSPIRE].

[64] R. Kleiss and H. Kuijf, Multi-Gluon Cross-sections and Five Jet Production at Hadron Colliders, Nucl. Phys. B 312 (1989) 616 [InSPIRE].

[65] V. Del Duca, A. Frizzo and F. Maltoni, Factorization of tree QCD amplitudes in the high-energy limit and in the collinear limit, Nucl. Phys. B 568 (2000) 211 [hep-ph/9909464] [INSPIRE].

[66] V. Del Duca, L.J. Dixon and F. Maltoni, New color decompositions for gauge amplitudes at tree and loop level, Nucl. Phys. B 571 (2000) 51 [hep-ph/9910563] [INSPIRE].

[67] Z. Bern and Y.-t. Huang, Basics of Generalized Unitarity, J. Phys. A 44 (2011) 454003 [arXiv:1103.1869] [INSPIRE].

[68] F.A. Berends and W.T. Giele, Recursive Calculations for Processes with n Gluons, Nucl. Phys. B 306 (1988) 759 [INSPIRE].

[69] F. Cachazo, P. Svrček and E. Witten, MHV vertices and tree amplitudes in gauge theory, JHEP 09 (2004) 006 [hep-th/0403047] [INSPIRE].

[70] R. Britto, F. Cachazo and B. Feng, New recursion relations for tree amplitudes of gluons, Nucl. Phys. B 715 (2005) 499 [hep-th/0412308] [INSPIRE].

[71] R. Britto, F. Cachazo, B. Feng and E. Witten, Direct proof of tree-level recursion relation in Yang-Mills theory, Phys. Rev. Lett. 94 (2005) 181602 [hep-th/0501052] [INSPIRE].

[72] S. Badger, H. Frellesvig and Y. Zhang, Multi-loop Integrand Reduction with Computational Algebraic Geometry, J. Phys. Conf. Ser. 523 (2014) 012061 [arXiv:1310.4445] [INSPIRE].

[73] Z. Bern, L.J. Dixon and D.A. Kosower, A two loop four gluon helicity amplitude in $Q C D$, JHEP 01 (2000) 027 [hep-ph/0001001] [INSPIRE]. 
[74] Z. Bern, A. De Freitas and L.J. Dixon, Two loop helicity amplitudes for quark gluon scattering in QCD and gluino gluon scattering in supersymmetric Yang-Mills theory, JHEP 06 (2003) 028 [Erratum ibid. 04 (2014) 112] [hep-ph/0304168] [INSPIRE].

[75] G. 't Hooft and M.J.G. Veltman, Regularization and Renormalization of Gauge Fields, Nucl. Phys. B 44 (1972) 189 [inSPIRE].

[76] Z. Bern, A. De Freitas, L.J. Dixon and H.L. Wong, Supersymmetric regularization, two loop QCD amplitudes and coupling shifts, Phys. Rev. D 66 (2002) 085002 [hep-ph/0202271] [INSPIRE].

[77] Z. Bern, J.J.M. Carrasco and H. Johansson, New Relations for Gauge-Theory Amplitudes, Phys. Rev. D 78 (2008) 085011 [arXiv: 0805.3993] [InSPIRE].

[78] Z. Bern and D.A. Kosower, Color decomposition of one loop amplitudes in gauge theories, Nucl. Phys. B 362 (1991) 389 [inSPIRE].

[79] Z. Bern, J.J.M. Carrasco, L.J. Dixon, H. Johansson, D.A. Kosower and R. Roiban, Three-Loop Superfiniteness of $N=8$ Supergravity, Phys. Rev. Lett. 98 (2007) 161303 [hep-th/0702112] [INSPIRE].

[80] Z. Bern, J.J.M. Carrasco, L.J. Dixon, H. Johansson and R. Roiban, Manifest Ultraviolet Behavior for the Three-Loop Four-Point Amplitude of $N=8$ Supergravity, Phys. Rev. D 78 (2008) 105019 [arXiv:0808.4112] [INSPIRE].

[81] Z. Bern, J.J.M. Carrasco and H. Johansson, Perturbative Quantum Gravity as a Double Copy of Gauge Theory, Phys. Rev. Lett. 105 (2010) 061602 [arXiv:1004.0476] [INSPIRE].

[82] Z. Bern, J.J.M. Carrasco, L.J. Dixon, H. Johansson and R. Roiban, The Complete Four-Loop Four-Point Amplitude in $N=4$ super-Yang-Mills Theory, Phys. Rev. D 82 (2010) 125040 [arXiv:1008.3327] [inSPIRE].

[83] Z. Bern, J.J.M. Carrasco, L.J. Dixon, H. Johansson and R. Roiban, Simplifying Multiloop Integrands and Ultraviolet Divergences of Gauge Theory and Gravity Amplitudes, Phys. Rev. D 85 (2012) 105014 [arXiv: 1201.5366] [INSPIRE].

[84] Z. Bern, E. Herrmann, S. Litsey, J. Stankowicz and J. Trnka, Logarithmic Singularities and Maximally Supersymmetric Amplitudes, JHEP 06 (2015) 202 [arXiv:1412.8584] [INSPIRE].

[85] Z. Bern, E. Herrmann, S. Litsey, J. Stankowicz and J. Trnka, Evidence for a Nonplanar Amplituhedron, JHEP 06 (2016) 098 [arXiv: 1512.08591] [INSPIRE].

[86] J.M. Henn and B. Mistlberger, Four-Gluon Scattering at Three Loops, Infrared Structure and the Regge Limit, Phys. Rev. Lett. 117 (2016) 171601 [arXiv:1608.00850] [INSPIRE].

[87] S. Franco, D. Galloni and A. Mariotti, The Geometry of On-Shell Diagrams, JHEP 08 (2014) 038 [arXiv: 1310.3820] [inSPIRE].

[88] B. Chen, G. Chen, Y.-K.E. Cheung, Y. Li, R. Xie and Y. Xin, Nonplanar On-shell Diagrams and Leading Singularities of Scattering Amplitudes, Eur. Phys. J. C 77 (2017) 80 [arXiv:1411.3889] [INSPIRE].

[89] N. Arkani-Hamed, J.L. Bourjaily, F. Cachazo, A. Postnikov and J. Trnka, On-Shell Structures of MHV Amplitudes Beyond the Planar Limit, JHEP 06 (2015) 179 [arXiv: 1412.8475] [INSPIRE].

[90] S. Franco, D. Galloni, B. Penante and C. Wen, Non-Planar On-Shell Diagrams, JHEP 06 (2015) 199 [arXiv: 1502.02034] [InSPIRE]. 
[91] B. Chen, G. Chen, Y.-K.E. Cheung, R. Xie and Y. Xin, Top-forms of Leading Singularities in Nonplanar Multi-loop Amplitudes, arXiv:1506.02880 [INSPIRE].

[92] J.L. Bourjaily, S. Franco, D. Galloni and C. Wen, Stratifying On-Shell Cluster Varieties: the Geometry of Non-Planar On-Shell Diagrams, JHEP 10 (2016) 003 [arXiv:1607.01781] [INSPIRE].

[93] N. Arkani-Hamed, J.L. Bourjaily, F. Cachazo, A.B. Goncharov, A. Postnikov and J. Trnka, Scattering Amplitudes and the Positive Grassmannian, Cambridge University Press (2012).

[94] N. Arkani-Hamed and J. Trnka, The Amplituhedron, JHEP 10 (2014) 030 [arXiv: 1312.2007] [INSPIRE].

[95] M.L. Mangano, The Color Structure of Gluon Emission, Nucl. Phys. B 309 (1988) 461 [INSPIRE].

[96] M.L. Mangano and S.J. Parke, Multiparton amplitudes in gauge theories, Phys. Rept. 200 (1991) 301 [hep-th/0509223] [INSPIRE].

[97] F. Maltoni, K. Paul, T. Stelzer and S. Willenbrock, Color flow decomposition of QCD amplitudes, Phys. Rev. D 67 (2003) 014026 [hep-ph/0209271] [INSPIRE].

[98] H. Ita and K. Ozeren, Colour Decompositions of Multi-quark One-loop QCD Amplitudes, JHEP 02 (2012) 118 [arXiv:1111.4193] [INSPIRE].

[99] C. Reuschle and S. Weinzierl, Decomposition of one-loop QCD amplitudes into primitive amplitudes based on shuffle relations, Phys. Rev. D 88 (2013) 105020 [arXiv:1310.0413] [inSPIRE].

[100] T. Schuster, Color ordering in QCD, Phys. Rev. D 89 (2014) 105022 [arXiv:1311.6296] [INSPIRE].

[101] T. Melia, Dyck words and multiquark primitive amplitudes, Phys. Rev. D 88 (2013) 014020 [arXiv:1304.7809] [INSPIRE].

[102] T. Melia, Getting more flavor out of one-flavor QCD, Phys. Rev. D 89 (2014) 074012 [arXiv: 1312.0599] [INSPIRE].

[103] T. Melia, Dyck words and multi-quark amplitudes, PoS (RADCOR 2013) 031.

[104] H. Johansson and A. Ochirov, Color-Kinematics Duality for QCD Amplitudes, JHEP 01 (2016) 170 [arXiv: 1507.00332] [InSPIRE].

[105] T. Melia, Proof of a new colour decomposition for QCD amplitudes, JHEP 12 (2015) 107 [arXiv: 1509.03297] [INSPIRE].

[106] T. Ohl, Drawing Feynman diagrams with Latex and Metafont, Comput. Phys. Commun. 90 (1995) 340 [hep-ph/9505351] [INSPIRE]. 